\title{
TITLE: ON EXTENDED QUANTUM-HOLOGRAPHIC FRAMEWORK FOR CONSCIOUSNESS AND FREE WILL: ROUND TRIP FROM SCIENCE TO SPIRITUALITY
}

\author{
Author: Dejan Raković \\ Affiliations: \\ Faculty of Electrical Engineering, University of Belgrade, Serbia
}

\begin{abstract}
The subject of this papar is our extended quantum-informational theoretical framework for consciousness and free will. The presented framework might have significant epistemological-cognitive and psychosomatic-spiritual implications, providing better understanding of transpersonal origin of quantuminformational psychosomatic processes in integrative medicine and transpersonal psychology. The paper also provides two appendices: the first one reviews what a psychologist needs to know on our extended quantumholographic / quantum-gravitational informatics, while the other one presents physical and informational extended glossary for more interested readers.
\end{abstract}

Keywords: Extended Hopfield-like quantum-informational theoretical framework, consciousness, free will, integrative medicine, transpersonal psychology.

\section{Introduction}

Consciousness has been the central theme of philosophical essays for a long time from the very beginning of philosophical thought, or traditional esoteric practices of the East and West which have reached significant level in control of altered states of consciousness with significant philosophical-religious implications.

The first scientific attempts to enlighten the nature of consciousness appeared only in psychology of the second half of 19th century (through development of psychophysics and theories of personality, and afterwards in $20^{\text {th }}$ century through attempts to establish controversial experimental parapsychology, which however have contributed to holistic climate for development of transpersonal psychology and integrative medicine in recent decades [1-3]), in physics of the beginning of 2oth century (through development of quantum mechanics and articulation of the problem of the so called wave function reduction, and the role of observer in that process), and in computer sciences of the second part of 2oth century (through development of artificial intelligence and the conception that the whole cognitive process can be reduced to a computer algorithm). In past two decades Center for Consciousness Studies of the University of Arizona in Tucson is organizing annual multidisciplinary conferences on consciousness [4], as research on the nature of consciousness comes again in the focus of the world scientific community because of potential implications in many scientific fields. ${ }^{2}$

\footnotetext{
${ }^{1}$ Author's email: www.dejanrakovic.com rakovicd@etf.bg.ac.rs

${ }^{2}$ For instance [5], in medicine, it might give rise to enlightening of the role of acupuncture system and consciousness in development of psychosomatic diseases and their prevention and healing. In psychology, we could understand mechanisms and roles of altered states of consciousness in the growth of personality, control of creativity, as well as
} 
The paper is organized as follows (with numerous more explaining footnotes throughout). Section 1 introduces the motivation and significance of consciousness studies. Section 2 considers the wave function collapse as a fundamental framework for searching physical origins of consciousness and free will. Sections 3 and 4 review epistemological-cognitive and psychosomatic-spiritual holistic implications of our extended quantum-holographic / quantum-gravitational (QHQG) theoretical framework of consciousness and free will. Section 5 discusses transpersonal global origins of trans-generation holistic programs and spiritual freedoms of their optimization. Section 6 concludes on necessity of global reprogramming of collective consciousness, with shift towards holistic gentle actions for solutions of the world risk society. There are also two appendices: Appendix A comprises information what a psychologist needs to know on our extended QHQG informatics, while Appendix B presents physical and informational extended glossary.

\section{Wave Function Collapse: On Consciousness and Free Will}

When we speak about quantum processes, the term quantum refers primarily to the discrete element of energy $E$ in the microsystem, to which the fundamental oscillation frequency $v$ is ascribed, according to the famous Planck formula $E=h v$, where $h$ is the Planck constant. This deep relation between discrete energy levels and oscillation frequencies lies in the basis of wave / particle duality, characteristic of quantum phenomena. The laws governing these microscopic quantum entities differ from those that govern the everyday classical world, for example, quantum particles can exist in multiple states or multiple locations simultaneously, where such multiple parallel quantum superposition alternatives (of which each alternative is measured by a complex number) would be described by a mathematical quantum wave function.

From the very beginnings of quantum mechanics, the so-called problem of measurement was recognized, as a question why we do not observe quantum superposition in macro-world as well, where we see objects and particles in various locations and in different states. More precisely, the problem of measurement is the conflict between two fundamental procedures in quantum mechanics. The first procedure relates to continuous deterministic evolution of the quantum state, known as unitary evolution (described by fundamental Schrödinger equation). The second procedure is applied when measurement on the quantum system or observing the quantum system is achieved, where the quantum state is discontinuously and probabilistically replaced by another quantum state, known as a state reduction or wave function collapse (described by von Neumann projection postulate) [6].

This conflict is marked as a problem of measurement (perhaps more precisely the paradox of measurement), and its problematic nature becomes manifest when we consider the measuring apparatus itself as a quantum entity, which is part of the entire quantum system consisting of the original observed system together with this measuring instrument. The apparatus is composed of the same quantum constituents (electrons, photons, protons, neutrons, etc.) as the observed system, and is subject to the same quantum laws and is described by continuous unitary evolution. How, then, can the discontinuous and probabilistic reduction result from the interaction (measurement) between the two parts of the quantum system? It is a paradox that basically indicates that quantum mechanics is still an incomplete theory. There are several attempts to solve this paradox, and some include different objective reductions (ORs) with a specific objective threshold that causes a quantum state reduction $[7,8]$.

transpersonal phenomena usually accompanying these states. In biology, it might appear that limits of interactions between individuals are more provisional than widely believed, which would be of significance not only for adaptive mechanisms on the level of the whole biological species, but even for deeper understanding of the very significance of morals in human population. In physics, an understanding of the nature of consciousness and free will could give rise to a deeper understanding of fundamental problems of the observer's role in quantum-mechanical act of measurement, which would demonstrate that consciousness, space, time, and matter are more deeply interwoven than widely believed. In communications, an understanding and controlling of transpersonal interactions might radically surpass many traditional barriers. In computer sciences, an understanding of the nature of consciousness could give rise to computers with artificial consciousness, which would function on deeper quantum-mechanical principles. Finally, in philosophy and religion, we might radically shift our scientific understanding of some ultimate questions, traditionally remaining outside the domain of theoretical and experimental scientific methods of natural sciences, being therefore a subject of deep and painful irrational divisions throughout the whole history of human civilization. 
On the other hand, the indeterministic characteristics of free will indicate that (related to it) consciousness must play an essential role in the wave function collapse [6-13], the only indeterministic property of quantum mechanics, which still has manifestly open problems of the physical nature of nonlinear collapse and relativistically inconsistent instantaneous distant action of the nonlocal collapse of the wave function [9-13].

One possible solution of the problem of (nonlinear) collapse is proposed by Penrose [8] in gravitationally induced OR of the wave function (superposition of quantum-entangled alternatives of quantum system (S) / measuring apparatus-environment (E)), in which the gravitational field of $E$ implies the superposition of different space-time geometries included, and when these geometries become sufficiently different (i.e. gravitational self-energy $E_{G}$ of the difference of mass distribution of the superimposed states) this implies the break of standard quantum-entangled superposition of the states S / E (ill-defined in strictly separate space-time geometries!) and Nature must choose between one of them and actually affects OR of the wave function for time $\tau \approx \hbar / E_{G}$ (inversely proportional to the size of quantum system $S$ ). ${ }^{3}$

According to our extended quantum-holographic / quantum-gravitational (QHQG) theoretical model of consciousness [9-12], the similar $O R$ of the wave function might have quantum-gravitational origin in microscopic space-time wormholes of highly noninertial microparticle interactions in quantum measurement-like situations (fully equivalent, according to Einstein's Principle of equivalence, to strong gravitational fields - in which opening of wormholes is predicted $[15,16])$. The question how it is possible that these highly non-inertial microparticle processes with inevitable relativistic generation of microparticles' wormholes were not taken into account within quantum mechanics which is yet extremely accurate theory(?) - might be answered as they were(!), but implicitly within the ad hoc von Neumann's projection postulate [6] to account for quantum mechanical $O R$ of the wave function in quantum measurement-like situations - implying also that von Neumann's ad hoc Projection postulate is based on quantum gravitational phenomena [9-12], on deeper physical level than nonrelativistic quantum mechanical ones: $4,5,6,7,8,9,10,11,12$

\begin{abstract}
${ }^{3}$ Regarding non-algorithmic quantum-gravitational aspects of consciousness [8], Hameroff and Penrose tried to search for sufficiently isolated relevant macroscopic quantum degrees of freedom in microtubular citoskeletal structures of neurons (thus avoiding ultra-short OR-based 'proto-conscious observations' of the stochastic environment!), in order to keep unitary evolution of the quantum superposition of microtubules until the time of orchestrated reduction (Orch OR) $\tau \approx \hbar / E_{G} \approx 25 \mathrm{~ms}$ (characteristic for $\gamma$-brainwaves in EEG, neurophysiologically correlated with consciousness), which might produce the moment of consciousness according to the Orch OR scheme [7] - which was undergone to severe critiques in Tegmark's paper [15].
\end{abstract}

${ }^{4}$ Such macroscopically accumulated exotic OR-induced anti-gravitational imprints might possibly be observable on cosmological scale (or have been already detected (?!) by recent advances in the cosmological tests, that invite again the Einstein's cosmological constant and related concept of hypothetical "dark energy" or "quintessence", which permeates all of space and tends to accelerate the expansion of the universe [17])!?

${ }^{5}$ In this context, it is expected that only on the level of future Quantum gravitation theory the nature of 'black holes', 'wormhole' tunnels, and 'cosmological singularities' will be revealed, when on the Planck-Wheeler's scales of length $1,62 \cdot 10^{-35} \mathrm{~m}$ and time $\sim 0,54 \cdot 10^{-43} \mathrm{~s}$ the concept of space-time ceases to exist and remains only Wheeler's 'quantum foam', out of which space-time singularities and surrounding parts of space-time are made [18].

${ }^{6}$ The relic of the aforementioned microscopic processes remained on the macroplane in the transitional states of individual consciousness (as highly non-inertial processes of the space-time quantum-gravitational mentally-channeled tunneling of the displaced part of the acupuncture EM-ionic quantum-holographic neural network), which can be a biophysical basis [9-12] of transpersonal communication [19-24] - in which mental addressing on the target implies unique ontological personal aspect of individual human consciousness [9-12], which is the widespread thesis of Christian religious tradition $[25,26]$.

${ }^{7}$ It should be noted that many transpersonal phenomena are even practically incorporated in the psychosomatic energy correction (cf. [3] and refs. therein) and that their physical explanation should be sought at the very border of the existing scientific paradigm. In our extended $Q H Q G$ theoretical framework of consciousness and psychosomatics [912], it is assumed that they are of deeper quantum-gravitational origin (in spatio-temporally transcending highly-noninertial strong-gravitation-equivalent (according to Einstein's principle of equivalence!) transitional states of consciousness (from high-dielectric body to low-dielectric out-of-body states!), based on locally generated wormhole space-time tunnels, quantum-gravitationally stabilized by so-called exotic matter (quantum vacuum fluctuations in strongly curved space-time wormhole tunnels [15]) with anti-gravitational effects, really observed in transpersonal psycho-kinetic manifestations of vital energy / prana / qi [21-24].

${ }^{8}$ The exotic aspect of wormhole tunnels and vital energy might be extended to acupuncture channels (with vacuum-like 
outside inflow of vital energy / prana / qi) - implying that such type of exotic transpersonal communication enables continuously-refreshing interaction between the two mentally-addressed persons [27]. This transpersonally stabilizes quantum-entanglement of their acupuncture systems / consciousnesses i.e. prevents dis-entanglement of their macroscopic quantum-entangled non-stationary states by OR-like interaction with their local somatic environments (contrary to situation in microworld without such stabilizing mentally-addressing exotic transpersonal effects, where laboratory-created quantum-entangled pairs of particles must be well isolated from environment in order to prevent their subsequent quantum-decoherence)!

[Regarding the EM microwave (MW) experiments [28] with unusual vacuum-like $n=1$ refraction index of acupuncture channels - based on the principles of photonic crystals and photonic bandgap guiding (where the confinement of photons in some low-index hollow-core 3D-defect might be achieved by exploiting photonic bandgap reflectivity of the surrounding higher-index (quasi) periodic medium [29]!) - this inspired us to suggest an interesting possibility [30] to consider acupuncture channels as 3D photonic crystal channel waveguides of propagated EM MW photons (influencing backward the structure and ionic conductivity of the channels) - confined by the surrounding higher-index (quasi) periodic cellular body medium outside channels (of $n=5 \div 6$ in the cellular cytoplasm and $n \gg 1$ in the cellular membranes of metabolic depending striking polarization of the volume ion density [31]), with nonpropagated EM MW evanescent photons [32] pervading our body.]

${ }^{9}$ Additionally, if put in the theoretical context of the fundamental holographic principle [33,34], according to which any 3D physical system is isomorphic to the holographic information embedded on its 2D surface, then quantumholographic informational content of a body could also be contained in corresponding surface layer of vital energy of the astral-mental body (aura), as has been stated in various traditions - in accordance with our extended QHQG framework for psychosomatics and proposed close relationship of the acupuncture system and consciousness (supported by meridian psycho-energy therapies as well, with the rapid elimination of traumas, phobias, allergies and other psychosomatic disorders, by simultaneous emotional-involved intensive visualization of psychosomatic problems and tapping / touching on prescribed acupuncture points [10,35])! It was also conjectured [34] that volume itself is illusory and that Universe is really a hologram isomorphic to information "inscribed" on the surface of its boundary.

${ }^{10}$ Our extended QHQG theoretical framework might also imply that quantum-holographic hierarchical parts carry information about the whole (which resembles the Hindu relationship Brahman / Atman, as a whole and the part which contains information about the whole [10-12], allowing subtle fractal coupling of various hierarchical levels in Nature [36]. This might be supported by acupuncture system and its projection zones (ECIWO holographic concept [37]) and the discovery of quantum-holographic influence of verbal communication on the expression of cell genome (by voicemodulation of irradiating-laser beams [38]), as well as by the origin of the amazing creativities (Tesla and Mozart as case studies [11,12]) and predestined life paths and temptations (as indicated by the contemporary experiences of hypno-regressions [39] as well as experiences of different traditions [19], while still emphasizing that it is possible to optimize them by spiritual purification).

${ }^{11}$ According to our extended $Q H Q G$ framework [9-12], transpersonal interactions might be interpreted by OR-related consciousness-channeled quantum-gravitational tunneling of operator's individual consciousness - mentally addressed on the target's content of the collective consciousness / environment in operator's transitional states of consciousness (short-lasting and therefore poorly reproducible!) - thus intentionally channeling state of operator's individual consciousness: $|\Phi\rangle_{S} \rightarrow\left|\Phi_{j}\right\rangle_{S}$ and automatically influencing the complement collective consciousness / environment output: $|\Psi\rangle_{E} \rightarrow\left|\Psi_{j}\right\rangle_{E}$ in quantum-gravitationally-induced and consciousness-channeled OR:

$$
|\Phi\rangle_{S}|\Psi\rangle_{E} \rightarrow \sum_{i} c_{i}\left|\Phi_{i}\right\rangle_{S}\left|\Psi_{i}\right\rangle_{E} \stackrel{p_{j}=\left|c_{j}\right|^{2} \approx 1}{\longrightarrow}\left|\Phi_{j}\right\rangle_{S}\left|\Psi_{j}\right\rangle_{E}
$$

The above relation is basically description of quantum entanglement between memory attractors of individual consciousness $\left|\Phi_{i}\right\rangle_{S}$ and collective consciousness $\left|\Psi_{i}\right\rangle_{E}$ (quantum-correlated via consciousness-based resonant recognition / interaction of associatively-related attractors of individual and collective consciousness), followed by body-induced OR-related stochastic process (with previously mentally enhanced $\left|c_{j}\right| \approx 1$ contribution by focusing on $\left|\Phi_{j}\right\rangle_{s}$ memory attractor of individual consciousnes, cf. Fig. 1(a)). In this respect, the "gauge quantum field theory and quantum gravity correspondence" of any quantum entangled system (i.e. Einstein-Podolsky-Rosen correlated quantum pair) and corresponding connected miniature (non-traversable) wormhole (i.e. quantum-gravitational Einstein-Rosen bridge) [16] should be pointed out - as note added in proof of our extended $Q H Q G$ framework for transpersonal interactions via miniature wormholes (on their possible traversability in cosmological conditions see ref. [15], while in transpersonal conditions see refs. [9-12]). 
On the other hand, nonlocality of collective consciousness [8-12], as a giant space-time associative neural network with distributed individual consciousnesses (related to bodily acupuncture MW ULF-modulated quantumholographic neural networks, mutually interacting quantum-gravitationally in transitional states of individual consciousnesses), might explain (apparently) instantaneous action in (nonlinear) quantum-gravitationally induced and (nonlocally) channeled OR by field-related collective consciousness. At the same time, the analogy of mathematical formalities of Hopfield's associative neural network and Feynman's propagator version of Schrödinger's equation [41] - points to collective consciousness as a possible ontological property of the physical field itself [8-12] ${ }^{13}$ with different microquantum and macro-quantum excitations (nonbiological and biological, real and vitual), which is widely spreaded thesis of Eastern traditions [19-24].

\section{Epistemological-Cognitive Implications}

Our extended QHQG approach to consciousness and psychosomatics also implies two modes of knowledge (as

[Note that the above expression also describes a much more frequent, constantly fluctuating, spontaneous interaction of individual consciousness with a closer environment, making that with sensory-focused contents we have the experience of reality as in normal states of consciousness! This continuous fluctuation has its analogy in the quantum biomolecular world, between continuously fluctuating non-complementary "states of biomolecular energy" and "states of biomolecular conformation" (and entangling "states of biomolecular environment") [40]!]

${ }^{12}$ It should be pointed out, that in the context of necessary conditions for decoherence described by the expression in the footnote above, defining of open quantum system and its environment is - simultaneous process - so that in the context of universal validity of quantum mechanics consciousness is relative concept, non-locally also influenced by farther parts of existing observing universe (and vice versa!), simultaneously creating conditions for the process of decoherence in the context of existing relative borderline [9-11]:

$$
\left.\left.|\Phi\rangle_{S}|\Psi\rangle_{E} \equiv \mid(\text { partial }) \text { individual / collective consciousness }\right\rangle_{S} \mid(\text { complement }) \text { environment }\right\rangle_{E} .
$$

This is fully in accordance with the idea of collective consciousness as a possible ontological property of the physical field itself, with different micro-quantum and macro-quantum (both non-biological and biological, real and virtual) excitations. Then, as cosmic collective consciousness (coincident with the "field" of Universe) has its complement "particle" cosmic environment, their strong-interaction-coupling affects decoherence of the "field" of cosmic collective consciousness into stationary classically-reduced (observing) stochastic state, with probabilities of realizations of corresponding classicaly-reduced states of cosmic collective cosnsciousness.

[However, note that the cosmic composite quantum state:

$$
\left.\left.|\Phi\rangle_{S}|\Psi\rangle_{E} \equiv \mid \text { cosmic "field" collective consciousness }\right\rangle_{S} \mid \text { (complement) cosmic "particle" environment }\right\rangle_{E}
$$

evolves without collapse (due to absence of the complement outside-cosmic environment!), which implies that Universe as a whole is presumably quantum hologram subject to deterministic Schrödinger evolution (in the context of universal validity of quantum mechanics!).]

${ }^{13}$ According to direct mathematical correspondence between classical neural variables (left) and quantum variables (right): $\boldsymbol{q} \Leftrightarrow \phi, \boldsymbol{q}^{k} \Leftrightarrow \phi^{k}, J \Leftrightarrow G$, and corresponding Hopfield's classical and Feynman's quantum equations [10,41], it might be realized that quantum memory attractors $\phi^{k_{i}}(r, t)$ of (any!) quantum system behave as quantum analogues of classical neural activities in space $r$ and time $t$ - implying that wave functions $\phi^{k_{i}}(i=1, \ldots, P)$ at space-time points $(r, t)$ are behaving fundamentally as $P$-dimensional parallel neural quantum processors (notice also their difference in respect to usually exploited 2-dimensional qubits in quantum informatics).

[This allows successive reconstruction of the wave functions of the memory states at the output of quantumholographic Hopfield-like neural network (complete, of both amplitude and phase) in recognition of the wave function of the previously memorized state displayed again at its input (which is the basis of every holography, but everything here is simpler than in the standard laser holography, which requires so-called coherent reference $\&$ object laser beams). It should be added that practical requirements for 2-qubit quantum computers are very demanding, with low tolerance $(\sim 0.1 \%)$, which will require operation at very low temperatures $(\sim \mu \mathrm{K})$ to eliminate thermal noise, as well as high degree of isolation from environments to eliminate the effects of decoherence. Due to its mode of operation (where the decoherence by the environment is included in their very information processing), the quantum-holographic Hopfieldlike neural networks are significantly more robust and more resistant to the above requirements - with possible operation at room temperature (like biological acupuncture system / consciousness)!] 
preserved in many traditions!), according to coupling strength consciousness-body-environment [10-12,27]: (i) strongly-coupled indirect mode (in bodily perceptively-rationally mediated classically-reduced stationary normal states of consciousness, like sensory perception, logic and scientific reasoning...); and (ii) weakly-coupled direct mode (in outof-body spiritually-creative quantum-entangled non-stationary transitional states of consciousness / quantum-coherent stationary altered states of consciousness, like prayer, meditation, daydreams, lucid dreams, deep creative insights...)with conditions of mutual transformation [10-12] (cf. also Fig. 1 and footnote 18). ${ }^{14,15,16,17}$

${ }^{14}$ This implies the generally poor informational rationalization of any direct quantum-holographic spiritual / religious
mystical experience (as OR-like collapse of the implicate order of quantum-entangled (quantum-holographic)
superposition of states of individual and collective consciousness into the explicate order of observed classically-
reduced states of individual and collective consciousness) - which differ from tradition to tradition! On the other hand,
the consensuses of the mystics of the same tradition of a similar partial rationalization of mystical experiences could be
understood as part of the same dominant-channeling trans-generational contextual heritage of members of the same
tradition (because we are not born as so-called tabula rasa, as being indicated by contemporary experiences of hypno-
regressions and experiences of different traditions as well!). [So science is closing the circle, by re-discovering two different modes of knowledge and at the same time setting its own epistemological limitations [10-12] - as preserved for millennia in shamanistic tribal traditions, which consider that true reality is represented by lucid dreams, and that the waking state is an illusion [20,21], or as concisely described by Patanjali in Yoga Sutras more than two millennia ago, pointing out that mystical experience (samadhi) is "filled with truth" and that it is "above inference and the scriptures" [42], or as formulated at the beginning of the last century by Berdyaev in Philosophy of Freedom as the difference of two modes of knowledge, prayer-mediated "comprehension of affairs invisible" and rationally-mediated "comprehension of affairs visible" [43]!]

${ }^{15}$ In addition, necessity of direct quantum-holographic coupling of individual and cosmic collective consciousness in an observation of the implicate order might need weak out-of-body quantum-communication coupling consciousnessenvironment, i.e. previous reprogramming of all psychosomatic loads (cleansing of the possessive or hedonistic emotional-mental sinful / karmic connections with world - which as loading "mental addresses" would give rise to quantum projections of the mentally-channelled tunnelled consciousness upon the out-of-body environment, and thus to classically-reduced out-of-body stationary extrasensory observing of the mentally-addressed environment). In this context the efforts of mystics of all traditions to purify consciousness / soul via spiritual practice (prayer, meditation...) and thus to reach their final eschatological goal (Kingdom of God, nirvana...), i.e. post-mortem salvation of soul (nonbounded sin-free / karmic-free) by re-union (re-ligare / yoga) with the supreme God [23] might appear reasonable!

[But it still would leave room for personal love, whose highest manifestation is ability and readiness for permanent and unconditioned forgiving (to loving person, and because of him / her to everybody else, including enemies!), in spiritual prayer and (subtly related quantum-holographic) living practice; the same refers to love for relatives and friends [10,11].]

${ }^{16}$ A nice example of the description of two modes of knowledge is also found in Mexican Jaki tradition [44], as two types of reality, tonal (sensory-perceived in the normal state of consciousness) and nagual (extrasensory-perceived in altered states of consciousness, with "silver ribbons" of vital energy that transpersonally connect us with our environment). Something similar is also mentioned in African-Haitian voodoo tradition (in which visualization deliberately can create a "silver ribbon" between the operator and the victim) [45] - which naturally exists between mother and child, and is spontaneously created by the intensive exchange of vital energy between relatives, close associates, current and former lovers, friends and enemies, and even post mortem can survive between living and deceased persons).

${ }^{17}$ It should be added that transpersonal esoteric concepts of different spiritual traditions [23] - might be accounted within our extended QHQG approach (with elaborated psychosomatic relationship consciousness / acupuncture macroscopic quantum-holographic Hopfield-like EM-ionic-exotic associative neural network [10,11,27]): (i) astral body (manomaya, lingasarira, manovijnana, ka, psyche, nephesh, nafs, subtle body, psychic body, soul...) - versus ionic component of the out-of-body displaceable EM-ionic macro-quantum acupuncture system (connected with the body by miniature "wormhole" space-time tunnel, generated in the highly-non-inertial transitional states of consciousness, as frequently observed in non-stationary extrasensory near-death experiences); (ii) mental body (vijnanamaya, suksmasarira, manas, ba, thymos, ruach, ruh, noetic body, spiritual body, mind...) - versus EM component of the outof-body displaceable EM-ionic macro-quantum acupuncture system (embedded within ionic component of the out-ofbody displaceable EM-ionic macro-quantum acupuncture system); (iii) distributed centers of consciousness (chakras, acupuncture points, sephiroths, holy discs...) - versus EM-ionic condensations in the structured out-of-body displaceable EM-ionic macro-quantum acupuncture system; (iv) vital energy (chi / qi / ki, prana / akasha, mana, ka, pneuma / ether / natura medica / anima mundi, yesod, baraka, the Holy Spirit / Uncreated Light, Heilstrom, Innate Intelligence, bioenergy / biofield...) - versus EM-ionic-exotic macro-quantum condensates (unstructured / structured, virtual / real) within acupuncture channels (with possible generalization from the EM field to the unified field, in line 


\section{Tesla's Inspired Holistic Ideas}

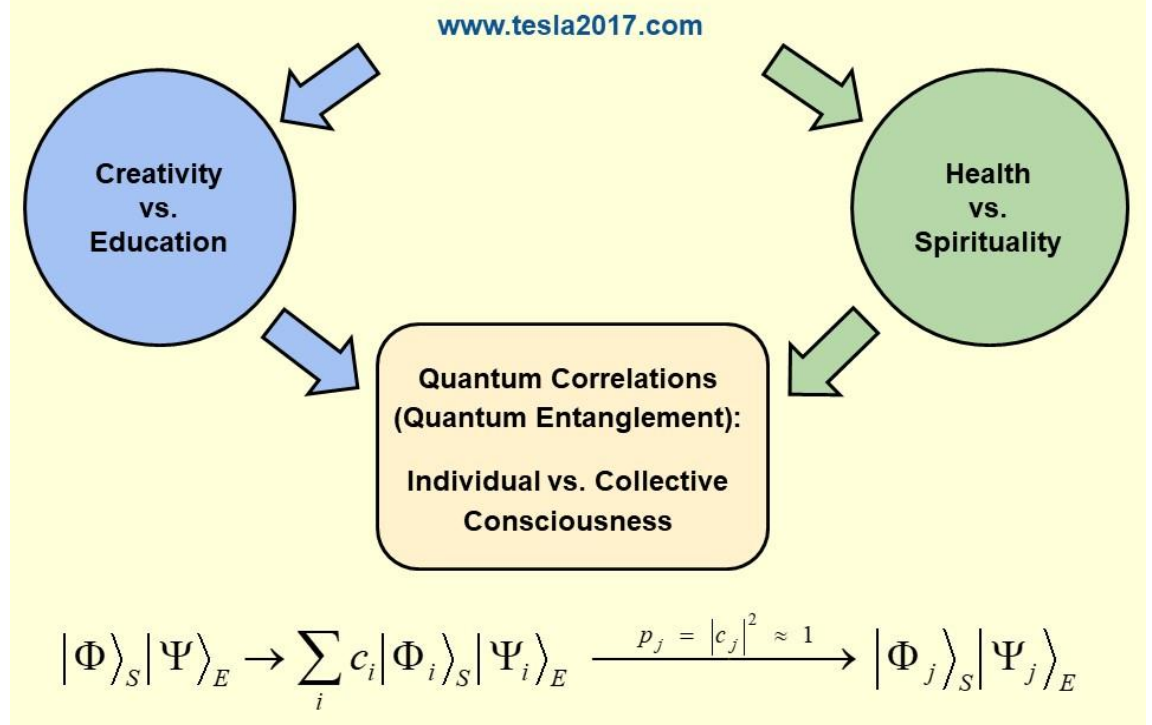

Figure 1. (a) Symbolic representation of mentally controlled-directed increasing of macro-quantum correlations of individual and collective consciousness in transpersonally-entangling creative phases (left-hand side of the image and formula above, with previous mental increase of the focused weight contribution $c_{j}$ and subsequent (practically non-stochastic) classically-reduced selection of this dominant weight contribution $c_{j}$ ) [11,12]; and (b) Symbolic representation of mentally controlled-systemic decreasing of macro-quantum correlations of individual and collective consciousness in transpersonally-disentangling spiritual phases (right-hand side of the image and formula above, with previously systemic mental decreasing of all weight contributions $c_{i}$ and subsequent (stochastic) classically-reduced selection of the weight contribution $c_{j}$ ) $[11,12] .^{18}$

with the broader traditional meaning of vital energy, as all-pervasive cosmic quintessence).

[Aforementioned consensus on the transpersonal esoteric concepts in different spiritual traditions, demonstrates that acquired information in their transpersonal extrasensory perceptions may give rise to non-distorting OR-like stationary extrasensory perception of their mentally-addressed out-of-body environments of collective consciousness (in Teslalike creative way [10-12]!) - in contrast to non-stationary mystical states of consciousness (with distorting OR-like nonstationary collapse of the implicate order of quantum-entangled (quantum-holographic) superposition of states of individual and collective consciousness into the explicate order of observed classically-reduced states of individual and collective consciousness).]

${ }^{18}$ In the context of creative insights and incredible Tesla inventions (directly visualized in his consciousness with the details of the functioning of the devices, without any application of Maxwell's electromagnetics!), it is interesting to consider the possibility of controlling creative processes within our extended QHQG theoretical framework [11,12]. They predict transpersonal properties of psyche in short-lasting quantum-entangled non-stationary transitional states of consciousness (with subsequent OR-reduced stationary extrasensory perception of the previously focused mentallyaddressed out-of-body complementary environment, which may also be Jung's "archetype" of the problem-withsolution at the level of quantum-holographic collective consciousness, which raises associations on Tesla's "mental inventions" and Plato's "world of ideas" as well). After returning of the displaced consciousness upon the body, in order that transpersonally acquired information ascends to the level of normally conscious state, it is necessary to overcome two filters: (i) acupuncture system / nervous system threshold filter (which requires "emotional colouring" of the solving problem) and (ii) frontolimbic amplification filter (which requires "emotional-thinking priority" of the solving problem).

[According to our investigations, all mentioned conditions might be realized in quantum-coherent stationary state of waking meditation (by entering in this prolonged altered state of consciousness, with mental addressing on the solved problem) - which Tesla has been doing by persistent mental focusing on the solving problem! On the other hand, during the sleep state the mentioned conditions might be realized in quantum-entangled non-stationary transitional states of falling asleep and quantum-coherent stationary states of REM-sleep (with previous intense concentration on the solving problem before sleep \& subsequent amplification of the obtained classically-reduced answer in the form of symbolic dream, which should be then correctly interpreted in the context of personal internal symbolism) - widely applicable 
This is in line with re-awakened interest in fundamental and applied holistic research in past decades, anticipating the occurrence of grand synthesis of two modes of knowledge in the framework of extended QHQG paradigm - where the role of each individual becomes indispensable due to influence and care for collective mental environment and social well-being.

And can help us realize that all our partial rationalizations (classically-reduced mappings!) are just approximations of fundamentally-holistic reality (quantum-holographic territory!), and that all divisions are tragic misunderstanding of spiritually immature epoch of civilization (as map is not the territory!).

All this indicates the full significance of holistic education and behavior - demonstrating ultimate necessity of redefining the global educational / informational / political goals, with the shift towards holistic gentle actions for global solutions of the world risk society.

\section{Psychosomatic-Spiritual Implications}

Holistic approach to integrative medicine and transpersonal psychology is of particular importance because of their ever-increasing use in developed countries [1-3] - since modern research into psychosomatic diseases indicates the need to apply holistic methods (with a focus on body's acupuncture system and consciousness [3,10-12]), oriented to treating a person as a whole (including mental environment), implying their macroscopic quantum origin. According to our extended QDQH approach, the healthy psychosomatic state (of (open) acupuncture system / consciousness) is considered as simplest informational state of the lowest quantum entropy (with single memory attractor), while disordered psychosomatic states are considered as more complex states of the higher quantum entropy (with additional side memory attractors, cf. Fig. 2).

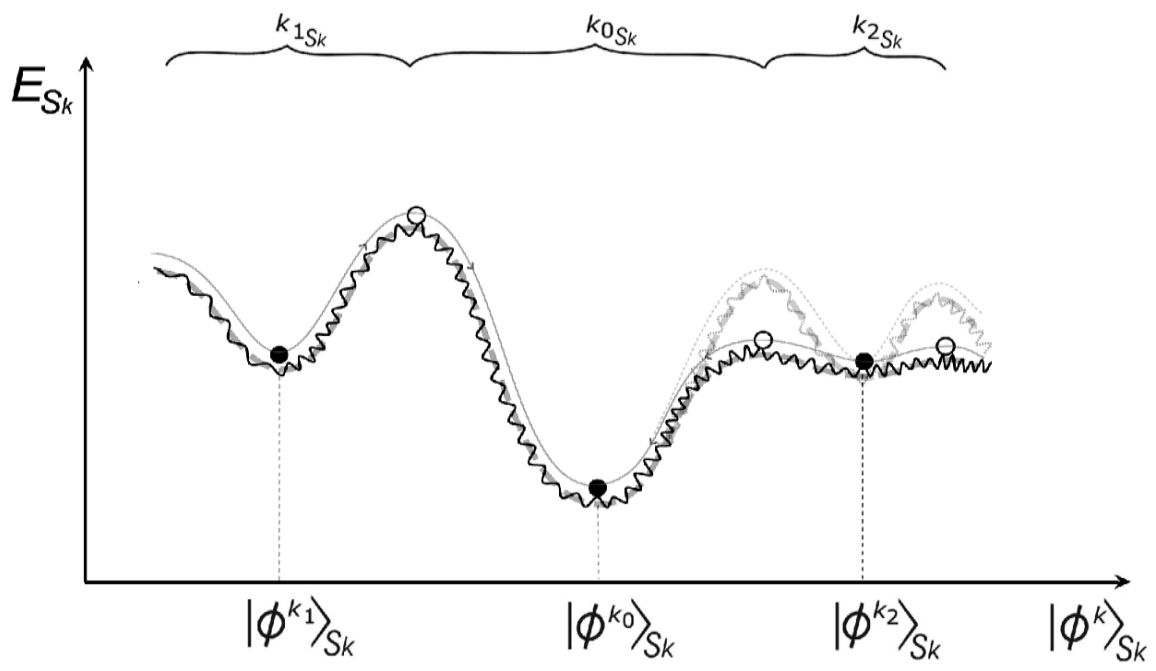

Figure 2. Schematic presentation of the adaptation of memory attractors in the energy-state hypersurface of the quantum-holographic memory / propagator of various hierarchical levels of biological macroscopic open quantum system(s) $S_{k}[10,11,46]$ (local cell's biomolecular protein / target, local acupuncture system / consciousness, or nonlocal out-of-body consciousness / collective consciousness). This implies necessity of quantum-informational successive bioresonant balansing of all unwilling side memory attractors (like $\phi^{k_{2}}$ in Fig.

even to meditation-non-trained persons! Of course, for solving conceptually complex scientific problems it is necessary to be an expert in the corresponding field, in order to scientifically rationalise a solution representing scientific shift.]

[The same might be applied to artistic creative experiences and their subsequent artistic expressions (Mozart as an excellent example!), while very art-works then behave like mental addresses of "archetypes" being in contact with artists in mentally-addressed transpersonal communications during the acts of creation. Then deep artistic experiences of the public might have strong spiritual note too, through spontaneous mental addressing of the artistic public on the masterpeace and emotionally-induced excitation into transitional state of consciousness. Finally, this could similarly be extended to deep spiritual experiences of the believers with their mental addressing on holy icon / relic \& emotionallyinduced excitation into transitional state of consciousness.] 
2), which would otherwise cause development of psychosomatic diseases on the individual level, and interpersonal fights in this and further generations on the collective level. ${ }^{19,20}$

Thus, all holistic acupuncture-based and consciousness-based approaches and techniques could be treated as quantum-informational therapies, by imposing new healing boundary conditions in the energy-state space of the (open) acupuncture system / (individual and collective) consciousness - implying three fronts lines of integrative medicine in top-down order, according to our extended QDQH approach to psychosomatics $[10,11,46]$ : (i) collective consciousness; ${ }^{21}$ (ii) acupuncture system / individual consciousness; ${ }^{22}$ and (iii) somatic level. It

${ }^{19}$ It should be noted that Nature presumably has chosen elegant room-temperature solution for quantum-holographic information processing, fluctuating between quantum-coherent and classically-reduced states of (open) biological macro-quantum system(s) $S_{\mathrm{k}}$, via non-stationary bioresonance interactions with out-of-body farther environment and decoherence by bodily closer environment - by adaptation of corresponding quantum-holographic memory / propagator $[10,11,46]$ (with time-adapting density of states of (open) macro-quantum system(s) $S_{k}$, represented by corresponding depths of minima like in Fig. 2):

$$
G\left(r_{2}, t_{2} ; r_{1}, t_{1}\right)=\sum_{i=1}^{P} \phi^{k_{i}}\left(r_{2}, t_{2}\right) \phi^{k_{i}^{*}}\left(r_{1}, t_{1}\right)=\sum_{i=1}^{P} A_{k_{i}}\left(r_{2}, t_{2}\right) A_{k_{i}}^{*}\left(r_{1}, t_{1}\right) e^{\frac{i}{\hbar}\left(\alpha_{k_{i}}\left(r_{2}, t_{2}\right)-\alpha_{k_{i}}\left(r_{1}, t_{1}\right)\right)}
$$

Thus quantum neural holography combined with quantum decoherence might be very significant element of the feedback bioinformatics, from the level of cell - to the level of organism - and further to the level of collective consciousness, with backward dynamic influence on the expression of genes.

${ }^{20}$ Further on, by considering psychosomatic state in generally mixed quantum state of density matrix:

$$
\widehat{\rho}_{S}=\sum_{i}\left|c_{k_{i}}\right|^{2}\left|\phi^{k_{i}}\right\rangle_{S}\left\langle\phi^{k_{i}}\left|\equiv \sum_{i} p_{k_{i}}\right| \phi^{k_{i}}\right\rangle_{S}\left\langle\phi^{k_{i}}\right|
$$

(where $p_{k_{i}}$ are probabilities of realization of the $k_{i}$-th quantum state), then von Neumann's quantum-mechanical entropy $S=-k_{B} \operatorname{Tr}\left(\hat{\rho}_{S} \ln \hat{\rho}_{S}\right)$ is reduced to classical Shannon's entropy $S=-k_{B} \sum_{i} p_{k_{i}} \ln p_{k_{i}}[10,11,46]$. So, within our exended $Q D Q H$ theoretical framework, entropy of pure-healthy state of the psychosomatic system (described by the single $k_{0}$-th term in the superposition, of probability $p_{k_{0}}=1$ ), would be $S_{k_{0}}=0$ (as pure quantum state $\hat{\rho}_{S_{k}}^{\left(k_{0}\right)}$ provides maximal possible information about the quantum psychosomatic system), while entropy of mixed-disordered state of the psychosomatic system (described by the full above superposition), would be $S_{\hat{\rho}}>0$ (as mixed stochastic state $\hat{\rho}_{S_{k v}}^{(k)}$ provides uncomplete information about the quantum psychosomatic system). So, we plausibly obtain that healthy psychosomatic state is the simplest informational state of the lowest quantum entropy (with single memory attractor), while disordered psychosomatic states are more complex states of the higher quantum entropy (with additional side memory attractors, cf. Fig. 2).

${ }^{21}$ Since there is no out-of-cosmic environment to push on non-unitary-OR a quantum-entangled state of the fieldrelated cosmic collective consciousness and the complementary 'particle' cosmic environment, the proposed mechanism of prayer (intrinsically non-unitary) sets the necessary new boundary conditions without mutual mentally-loaded addresses - so it can really be said that an all-forgiving prayer represents a biophysical basis for the optimization of (quantum) holistic global psychosomatics of collective consciousness (and complementary incoming individual and collective events) [10-12]! Let us add that both traditional and modern experiences of local and transpersonal energy healing [3] (by vacuum-excited spiritually-purifying new macro-quantum portions of vital energy and their subsequent mentally-channeled transfer), indicate that meditative visualization (intrinsically non-unitary) imposes new (OR-similar) quantum gravitational boundary conditions (but, in contrast to prayer, it would not eliminate simultaneously all mentally-loaded addresses, but would balance energy-informationally only one particular client. Therefore, in such situations, it is necessary to do circular meditative-healing visualization from all meta-positions involved in the existing conflict, thus eliminating simultaneously all mentally-loaded addresses)! Hence, in the context of our QDQH theoretical framework, spiritual prayer / meditation practices represent a non-unitary source of free will at the level of collective consciousness, which evolutionary increases the responsibility of every individual and the whole human race!

${ }^{22}$ The goal of these local acupuncture-based and consciousness-based procedures and approaches would be a bioresonant excitation of the psychosomatically disordered state (acupuncture palpatory-painful / psychologically traumatic) - thus enabling that its initial memory attractor is bioresonantly excited (similar to annealing procedure in neural networks [48]) becoming shallower and wider on the account of deepening of the (energy-dominating) attractor 
should be pointed out that necessary activities in the second and third levels, with neglect of the first level, would result in further transpersonal transfer of memory attractors on the level of individual and collective consciousness in this and future generations. So, this implies necessity to focus on the origins of many psychosomatic problems on underlying prenatal trans-generational levels (cf. [47] and refs. therein).

\section{On Trans-Generation Programs and Freedom of Optimization}

Michael Newton, a famous American transpersonal hypno-regression-therapist, writes about a number of psychotherapeutic experiences of post-hypnotic regressions, with systematized messages of his clients that our major life lessons and opportunities to optimize otherwise predestined life-programs are lying in spiritual sphere of purification [39]. Reading of Newton's book(s) brought me a special pleasure, due to many important associations and implications on correspondence of the described experiences and theoretical predictions of our extended QHQG framework [47]: (i) transpersonal-spiritual tunneling as exclusive communications, without space-time barriers (in accordance with our theoretical predictions); (ii) soul transpersonally chooses a pair of prospective parents, among several offered alternatives previously carefully observed with all details of a future life (indicating that life is predestined on the physical level by existing boundary conditions at the level of cosmic field / collective consciousness); (iii) by made choice of the parents, life is not completely fixed, but can be optimized by spiritual means, repenting and forgiving to ourselves and others (bringing new boundary conditions at the level of cosmic field / collective consciousness, which points to fundamental importance of spirituality as a source of the greatest freedom for optimizing otherwise predestined life-programs, and necessity of careful control of reactions on current life-temptations in order to avoid further deepening of the existing conflicts); (iv) although the aforementioned experiences evoke strong associations on reincarnation, some of the reported insights suggest the possibility of transmission of information of the same content into several persons during their overlapping lifetime periods (implying a trans-generational transfer of accumulated information, with experiences that transferred contents leave a strong impression of the previously lived lives).

So, in the context of aforementioned trans-generationally-predestined quantum-holographic structure of collective consciousness (as suggested by the experiences of tribal traditions, and traditions of the East and the West as well), it seems irrational not only to be angry at life program (previously freely chosen prenatally), but it is also irrational not to optimize it (spiritually-accepting and forgiving ourselves and our environment) contributing to both our own spiritual growth and catharsis of our trans-generational mental environment (which is fundamental question of both mental hygiene and civil decency i.e. both spiritual and civil morality)! $!^{23,24,25,26,27,28,29}$

of healthy (acupuncture palpatory painless / psychologically traumatic-free) state [10-12,46] - which is then altogether quantum-holographically projected on the lower quantum-holographic cellular level, thus changing expression of genes.

${ }^{23}$ It is hereby instructive to mention Buddhist interpretation of karma, which occurs not as a result of our spontaneous reaction to the current situation in life, but as a result of the latter justification of our conscious reactions against the others in this situation [49]! In the context of the above mentioned quantum-holographic predestined life programs, this is fully justified because we all (non-consciously) react to current conflicting-encoded life-situations (several hundred milliseconds before we become aware [50]!), but immediately afterwards we have a chance to (consciously) permanently untangle the existing trans-generational conflict by penitent-all-forgiving prayer. This is quite fair spiritual opportunity - and up to each person is either to use it or not - with accompanied either unloading or overloading trans-generational conflicts!

${ }^{24}$ That might be possibly the reason for Christianity to insist on repenting and forgiving ('And forgive us our debts, as we forgive our debtors', Mt.6,12), which represents a level of spiritual maturity of a person. This implies an obligation of spiritually mature persons to help in reprogramming mental / emotional conflicts by praying, even when themselves being victims of the conflicts ('But I say unto you, love your enemies, bless them that curse you, do good to them that hate you, and pray for them which despitefully use you, and persecute you', Mt.5,44). It is not even allowed to see evil in fellow-men and condemn them (an old biblical commandment given to the man in paradise, forbidding to take food 'of the tree of the knowledge of good and evil' - in fellow-men - 'for in the day that you eat there-of you shall die' (Gen.2,17), whose violation has caused the Adam's fall, and sowed the seed of the growing 'terror of history'), insisting only on personal repenting and forgiving, as the only way in permanent reprogramming of psychic conflicts. Anything else would enhance the existing mental / emotional personal loads and enlarge evil in the world, dismissing an individual and humankind from the final spiritual ideal of sinless state of consciousness - an old biblical commandment 


\section{Conclusion}

The above research is in line with awakened scientific interest in researching the nature of consciousness in past decades, with indication of the grand synthesis of two modes of knowledge - where the role of each individual becomes irreplaceable due to influence and care for collective mental environment - leaving the largest

after Adam's fall ("The man has now become like one of us, knowing good and evil. He must not be allowed to reach out his hand and take also from the tree of life and eat, and live forever." (Gen.3,22)).

${ }^{25}$ Bearing in mind the role of a mentally loaded contents in the post-mortem spiritual evolution of the soul of the dying (let us mention transpersonal collective levels of consciousness of Bardo levels in Tibetan tradition [51] or aerial toll house in Christian tradition [52]) - this implies importance of prayers for soul of the deceased with ability not only to influence its spiritual evolution, but also trans-generational preferences for individual and / or collective future offspring (with possibility of even retroactive-nonlocal trans-generational dis-entangling / un-binding of mental / emotional conflicts, as suggested by experiments with prayer [20] - bearing resemblance to quantum delayed choice experiments [53], and pointing additionally to quantum roots of consciousness)!

${ }^{26}$ We also mention some tribal traditions where initiated shamans ritually enter the altered states of consciousness and receive answers to the health problems of members of their tribes, and apply their healing methods based on reprogramming the loads on the level of tribes' collective consciousness (like in South America Ayahuaska tradition [54], or Nigerian Abiku tradition [55], with fascinating spiritual diagnosis and subsequent extension of the predestinedshort-life programs; or like in Hawaiian Ho'oponopono tradition [56], based on the participatory concept of taking full responsibility for any circumstance in which we find ourselves and repenting and forgiving ourselves in the context of disturbing life situations, with fascinating successes in reprogramming ourselves and our environment). In this context, I have to mention an incredible experience of my cousin Milica, with (epi) genetically conditioned high-risk pregnancy of very low level of amniotic fluid, normalized in just a few days of ho'oponopono prayer practices, with subsequent normal delivery!

${ }^{27}$ On the same line, we mention the contemporary spiritual teaching of Bruno Groening (1906-1959), the greatest faith healer of the 20th century, renown in Germany for biblical cases of miraculous mass healings during 1950s, whose fascinating divine healing channel is remained open after his death via spreading activities of his Circle of Friends all over the world [57]. Groening teaches that one can maintain health and heal from illness by absorption of a divine life force (germ. Heilstrom, most similar to Christian notion of Holy Spirit [23]) in upright position with arms and legs uncrossed and palms facing upwards, keeping strong faith in healing after mental request to Groening to take all his burdens! He never took credit for the healings, saying that he was only an instrument of God, regardless of persons' religion or nationality. Groening claimed that individual healing was only 5\% of his spiritual effects, while the rest 95\% was devoted to Great Reversal of humanity (this being very efficient way to optimize not only individual but also collective life programs!).

${ }^{28} \mathrm{We}$ also mention the fascinating world bestseller confession of Anita Moorjani [58] on spiritual changing her life program, in which she decided to change her previous life program (and thus forgive herself and her grieving husband) and to continue to live - with return from clinical death and after a few weeks even spontaneous healing of terminal phase of cancer; this greatly contributes to our understanding that even most difficult psychosomatic illness, like cancer, have their roots in the energy-information blockages and that healing begins by removing them - first lovingly to ourselves, accepting ourselves and forgiving ourselves, which is then manifested by love to others and acceptance of others (e.g. by spiritually dis-entangling / un-binding repentant-forgiving prayer [10-12,59])! See also [60] with more details on recommended holistic anti-stress approaches and techniques!

${ }^{29}$ Finally, we mention the experience of Jon RG (John-Richard - 50 years ago) Turner [61]: Some 50 years ago, I was living in Los Angeles, California. I was producing and hosting a talk show Holistic Viewpoints, Expanding Consciousness on KPFK-Pacifica Radio in Hollywood. At the same time, I had a psychotherapy practice in Beverly Hills. I was getting referrals of patients who had been declared incurable. Sitting at my marvelously carved Mexican desk, I closed my eyes and asked my Whole-Self what I could possibly do to assist these patients who had failed to find homeostasis after 5, 10, some even 20 years of various therapies. What entered my mind were the words "If you will take each person back to the period before they were born each will experience rapid healing!" With this instruction from my Whole-Self, I pioneered in reawakening and releasing the psychic memories each person had from before conception through after birth. What those patients discovered, in the first person is: Not only am I the synthesis of my parent's genetic DNA coding which gave me my physical characteristics, I am also the synthesis of their energetically charged thoughts and emotions during their pregnancy which gave me my mental and emotional characteristics: my Whole-Self emotional $D N A \subseteq(W-S e D N A \subset)$. The healing came from the recognition that the trauma feelings were the result of their mother's event not something they had done. 
space for free will and fundamentally enhancing significance of spiritual morality.

Thus civilization, via synthesis of scientific knowledge in the field of consciousness and millennia experiences of the spiritual traditions, could contribute that new millennium would be characterized by wisdom. Decisive role in this process could play all religious communities and spiritual practices, cultivating a true spiritual moral and absolute inter-personal, inter-ethnic and inter-religious tolerance, as a measure of individual behavior from the perspective of death, i.e. amount of generated and non-reprogrammed psychosomatic conflicts until death, which determines the spiritual evolution of the soul of the deceased as well as the preferences of the individual and / or collective future of the descendants - that can be significantly altered via collective prayer and acceptance of ourselves and our neighbors and enemies, and hence global reprogramming of collective consciousness.

This indicates full significance of holistic education and behavior - with necessity of redefining global educational / informational / political goals [62,63] - and shift towards holistic gentle actions for global solutions of the world risk society.

\section{Appendix A. What A Psychologist Needs To Know On Extended QHQG Informatics}

I recommend the book Holographic Universe to all interested in the phenomenology of various states of consciousness, with numerous well-documented mysterious phenomena in altered states of consciousness [21]. In this book, Michael Talbot relies heavily on the works of famous quantum physicist David Bohm and neurophysiologist Karl Pribram, who point out that the Universe could be a giant quantum hologram, the type of mind construct at the boundary of a subject / object - by relativizing the question of whether objective reality exists or constantly fluctuates between implicate and explicate orders? The book suggests that many well documented mysterious phenomena, like synchronicity and precognition, extrasensory perceptions and outof-body experiences, psychokinesis and teleportations, miraculous healing and mystical religious experiences could be explained within the (still controversial) holographic paradigm (cf. Glossaries B.1 - B.3). The reading and presentation of this book brought me great personal satisfaction, for my own research of quantum basis of consciousness and holistic psychosomatics and their implications $[10,12,46,47]$ (with numerous empirical / rational assertions, I recognized associatively by reading a book).

As an extraordinary case study, we mention Tesla's extraordinary creative experiences in his altered states of consciousness (categorized in his time as nonphysical phenomena) - with Tesla's conviction "when we start to study nonphysical phenomena, we will progress more in ten years than we have for centuries". In this context, it seems that Tesla's legacy for the new millennium is introspective evidence of his transpersonal creative communications [11], with fascinating possibility of his meditative eidetic control and increase of macroquantum correlations with the quantum-holographic primal source of collective consciousness (cf. Fig. 1(a) and Footnote 17). It could serve as a case study for understanding biophysical basis of both transpersonal psychology and control of creative processes, based on subtle interplay of normal and transitional and altered states of consciousness in meditation and sleep, and may help in developing future strategies for education [11,12]. These phenomenological insights could also serve as an additional confirmation of our extended QHQG theoretical framework of consciousness and psycho-somatics, based on the systemic reduction of quantum entropy and macro-quantum correlations between the acupuncture system / individual consciousness and collective consciousness (cf. Fig. 1(b)), which could help in developing future strategies for integrative medicine and transpersonal psychology; and in wider context, these insights could provide inspiration for rethinking global educational / informative / political goals - with reorientation towards holistic gentle actions for global solutions to world's risk society [11,12].

According to prevailing scientific paradigm, information processing within the central nervous system is occurring through hierarchically organized and interconnected neural networks [10]. It seems that this hierarchy of biological neural networks is going down sub-cellular cytoskeleton level, being according to some scientists a kind of interface between neural and quantum levels [7]. On the other hand, as any quantum system in Feynman's propagator version of quantum mechanics has formal mathematical structure of the quantumholographic Hopfield-like associative neural network [10,41] (cf. Footnote 12 and Glossaries B.4 - B.6) - then closely related macro-quantum acupuncture-based and consciousness-based memory attractors can be treated as psychosomatic states representing quantum holistic field records (cf. Fig. 2)! So, within our extended QHQC theoretical framework, the healthy psychosomatic state is the simplest informational state of the lowest quantum entropy (with single memory attractor), while disordered psychosomatic states as more complex states (with additional side memory attractors) of the higher quantum entropy (cf. Footnote 19 and Glossary 


\section{B.6).}

Then, according to our extended QHQG theoretical framework all holistic acupuncture-based and consciousness-based approaches and techniques could be treated as quantum-informational therapies, by imposing new bioresonantly exciting healing boundary conditions in the energy-state space of the (open) acupuncture system / (individual) consciousness. However, when this process is hindered by transpersonal entangled blockages in the energy-state space of EM field of the (open) acupuncture system / (individual and collective) consciousness - then memory attractors of quantum-holographic network of the field-related collective consciousness should be removed as well (via bioresonantly exciting prayer or circular psychotherapies from all relevant meta-positions included in the problem, to disentangle energyinformationally all mental addresses included in the problem, with final goal to achieve permanent healing i.e. spiritual integration of personality $[10,46])$. So, this implies necessity to focus on the origins of many psychosomatic problems on underlying prenatal trans-generational levels (cf. [47] and refs. therein).

Further on, according to our extended QHQG theoretical framework [10-12], physical explanation of transpersonal phenomena without space-time barriers (even practically incorporated in psychosomatic energycorrections [3]) - presumably might be sought in combination of quantum-gravitational-like transitional states of consciousness and Principle of equivalence and objective reduction (OR) of quantum wave function (cf. Eq. (1) in Footnote 10, and Glossary B.4). This relation is basically also description of quantum entanglement between memory attractors of individual $\left|\Phi_{i}\right\rangle_{S}$ and collective consciousness $\left|\Psi_{i}\right\rangle_{E}$ (quantum-correlated via consciousness-based resonant recognition / interaction of associatively-related attractors of individual and collective consciousness), followed by body-induced OR-related stochastic process (with previously mentally enhanced $\left|c_{j}\right| \approx 1$ contribution by focusing on $\left|\Phi_{j}\right\rangle_{S}$ memory attractor of individual consciousnes (cf. also Fig. 1(a)).

In this respect, the "gauge quantum field theory and quantum gravity correspondence" of any quantum entangled system (i.e. Einstein-Podolsky-Rosen correlated quantum pair) and created miniature (nontraversable) wormhole (i.e. quantum-gravitational Einstein-Rosen bridge) [16] should be pointed out (cf. Glossaries B.7 - B.10) - as note added in proof of our extended QHQG framework for transpersonal interactions via miniature wormholes (on their traversability in cosmological conditions see ref. [15], while in transpersonal conditions see refs. [10-12]). Note that Eq. (1) in Footnote 10 can also describe a much more frequent, constantly fluctuating, spontaneous interaction of individual consciousness with a closer environment, making that with sensory-focused contents we have the experience of reality as in normal states of consciousness (cf. also Glossary B.2). This continuous fluctuation has analogy in the quantum biomolecular world, between continuously fluctuating non-complementary states of biomolecular energy and states of biomolecular conformation (and entangling states of biomolecular environment) [40].

On the other hand, according to our extended QDQH theoretical framework [9,10], the similar highly-noninertial transitional processes should be expected in highly non-inertial microparticle interactions in quantummeasurement-like situations (fully equivalent, according to Einstein's Principle of equivalence, to strong gravitational fields - where openning of wormhole-tunnels is expected [15,16]). The question how it is possible that these highly non-inertial microparticle processes with inevitable relativistic generation of microparticles' wormholes were not taken into account within quantum mechanics which is yet extremely accurate theory(?) might be answered as they were(!), but implicitly within the ad hoc von Neumann's projection postulate [6] to account for quantum mechanical wave function $O R$ in measurement-like situations - implying also that von Neumann's ad hoc Projection postulate is based on quantum gravitational phenomena [9,10], on deeper physical level than nonrelativistic quantum mechanical ones!

On these lines, explanation of the long standing open problems of the physical nature of psychosomatic acupuncture system and consciousness, including exotic transpersonal aspects of vital energy (essentially important for our functioning according to Eastern traditional medicine!) - should presumably be sought on deeper quantum-gravitational level as well. The exotic aspect of wormhole tunnels and vital energy might be extended to acupuncture channels (with vacuum-like outside inflow of vital energy / prana / qi) - implying that such type of exotic transpersonal communication enables continuously-refreshing interaction between the two mentallyaddressed persons [27]. This transpersonally stabilizes quantum-entanglement of their acupuncture systems / consciousnesses i.e. prevents dis-entanglement of their macroscopic quantum-entangled non-stationary states by OR-like interaction with their local somatic environments (contrary to situation in microworld without such stabilizing mentally-addressing exotic transpersonal effects, where laboratory-created quantum-entangled 
pairs of particles must be well isolated from environment in order to prevent their subsequent quantumdecoherence)!

Additionally, if put in the theoretical context of the fundamental holographic principle [33,34] (cf. Glossary B.7), according to which any 3D physical system is isomorphic to the holographic information embedded on its 2D surface, then quantum-holographic informational content of $a$ body could also be contained in corresponding surface layer of vital energy of the astral-mental body (aura), as has been stated in various traditions (cf. Footnote 16) - in accordance with our extended QHQG framework for psychosomatics and proposed close relationship of the acupuncture system and consciousness (supported by meridian psycho-energy therapies as well, with the rapid elimination of traumas, phobias, allergies and other psychosomatic disorders, by simultaneous emotional-involved intensive visualization of psycho-somatic problems and tapping / touching on prescribed acupuncture points $[10,35])$ ! It was also conjectured [34] that volume itself is illusory and that Universe is a hologram isomorphic to information "inscribed" on the surface of its boundary.

Our extended QHQG theoretical framework might also imply that quantum-holographic hierarchical parts carry information about the whole (which resembles the Hindu relationship Brahman / Atman, as a whole and the part which contains information about the whole [10-12], allowing subtle fractal coupling of various hierarchical levels in Nature [36]. This might be supported by acupuncture system and its projection zones (ECIWO holographic concept [37]) and the discovery of quantum-holographic influence of verbal communication on the expression of cell genome (by voice-modulation of irradiating-laser beams [38]), as well as by the origin of the amazing creativities (Tesla and Mozart as case studies [11,12]) and predestined life paths and temptations (as indicated by the contemporary experiences of hypno-regressions [39] as well as experiences of different traditions [19], while still emphasizing that it is possible to optimize them by spiritual purification).

It should be also added, that in the context of necessary conditions for decoherence, defining of open quantum system and its environment is - simultaneous process - so that in the context of universal validity of quantum mechanics (cf. Glossary B.4) consciousness is relative concept, non-locally also influenced by farther parts of existing observing universe (and vice versa!), simultaneously creating conditions for the process of decoherence in the context of existing relative borderline [9] (cf. Eq. (2) in Footnote 11). This is fully in accordance with the idea of collective consciousness as a possible ontological property of the physical field itself, with different micro-quantum and macro-quantum excitations. Then, as cosmic collective consciousness (coincident with the "field" of Universe) has its complement "particle" cosmic environment, their stronginteraction-coupling affects decoherence of the "field" of cosmic collective consciousness into stationary classically-reduced (observing) stochastic state, with probabilities of realizations of corresponding classicalyreduced states of cosmic collective cosnsciousness. However, note that the cosmic composite quantum state of cosmic "field" collective consciousness and (complement) cosmic "particle" environment (cf. Eq. (3) in Footnote 11) evolves without collapse (due to absence of the complement outside-cosmic environment!), which implies that Universe as a whole is presumably quantum hologram subject to deterministic Schrödinger evolution (in the context of universal validity of quantum mechanics!).

So, in the context of the above proposed mechanism of prayer (which intrinsically non-unitary sets the necessary new boundary conditions without mutual mentally-loaded addresses, cf. Footnotes 20-24) - it can really be said that an all-forgiving prayer represents a biophysical basis for the optimization of (quantum) holistic global psychosomatics of collective consciousness (and complementary incoming individual and collective events) [10]! Let us add that both traditional and modern experiences of local and transpersonal energy healing [3] (by spiritually-excited and mentally-channeled new macro-quantum portions of vital energy and their subsequent mentally-channeled transfer), indicate that meditative visualization imposes new (decoherence-like) quantum gravitational boundary conditions (but, in contrast to prayer, it would not eliminate simultaneously all mentally-loaded addresses, but would balance energy-informationally only one particular client; therefore, in such situations, it is necessary to do circular meditative-healing visualization from all metapositions involved in the existing conflict, thus eliminating simultaneously all mentally-loaded addresses)!

Hence, in the context of our QDQH theoretical framework, spiritual prayer/meditation practices represent non-unitary source of free will at the level of collective consciousness, which evolutionary increases the responsibility of every individual and the whole human race [10-12]! It is hereby also instructive to mention Buddhist interpretation of karma, which occurs not as a result of our spontaneous reaction to the current situation in life, but as a result of the latter justification of our conscious reactions against the others in this situation [49]! In the context of the above mentioned quantum-holographic predestined life programs (and experiences of various traditions [19] and post-hypnotic regressions [39]), this is fully justified because we all 
(non-consciously) react to current conflicting-encoded life-situations (several hundred miliseconds before we become aware [50]!), but immediately afterwards we have a chance to (consciously) permanently untangle the existing trans-generational conflict by penitent-all-forgiving prayer. This might be possibly the reason for Christianity to insist on repenting and forgiving, as anything else would enhance the existing mental / emotional personal loads and enlarge evil in the world, dismissing an individual and humankind from the final spiritual ideal of sinless state of individual and collective consciousness!

In the context of trans-generational cosmic quantum hologram, although on the level of collective consciousness everything is quantum-holographically predestined - as suggested by experiences of different spiritual traditions [19] and post-hypnotic regressions [39] as well - they also suggest the possibility of optimizing life-programs by spiritual purification (cf. Footnotes 25-28 and Glossary G.1). In particular, let us mention Hawaiian ho'oponopono tradition with remarkable reprogramming of the life situations by contextual repenting and forgiving ourselves [56]; or South American ayahuaska and Nigerian Abiku traditions with fascinating spiritual diagnoses and extensions of the predestined-short-life programs [55]; or fascinating world bestseller of Anita Moorjani [58] on spiritual changing her life program with return from clinical death and after a few weeks spontaneous healing of terminal phase of cancer; or contemporary spiritual teaching of Bruno Groening with incredible healing channel remained open after his death [57], in optimizing both individual and collective life programs! This greatly contributes to our understanding that even most difficult psychosomatic disorders have their roots in the energy-information blockages, and that healing begins by removing them - first lovingly to ourselves, accepting ourselves and forgiving ourselves, which is then manifested by love to others and acceptance of others (e.g. by spiritually dis-entangling / un-binding repentant-forgiving prayer [10-12])!

\section{Appendix B. Physical \& Informational Extended Glossary}

\section{B.1. Altered States of Consciousness [Adopted from Wikipedia]}

Consciousness is the state or quality of awareness or of being aware of an external object or something within oneself. Recently, consciousness has also become a significant topic of interdisciplinary research in cognitive science, involving fields like psychology, linguistics, anthropology, neuropsychology and neuroscience. The primary focus is on understanding what it means biologically and psychologically for information to be present in consciousness i.e. on determining the neural and psychological correlates of consciousness. Issues of interest include phenomena such as subliminal perception, blindsight, denial of impairment, and altered states of consciousness produced by alcohol and other drugs, or spiritual or meditative techniques.

Altered state of consciousness (ASC) is any condition which is significantly different from a normal waking state. A first summary of the existing literature was carried out by Charles T. Tart in his book Altered States of Consciousness, which led to a more common use of the term. Tart analyzed a state of consciousness as made up of a number of component processes, including exteroception (sensing the external world); interoception (sensing the body); input-processing (seeing meaning); emotions; memory; time sense; sense of identity; evaluation and cognitive processing; motor output; and interaction with the environment. Each of these, in his view, could be altered in multiple ways by drugs or other manipulations. Research in this area has not yet reached firm conclusions, but a recent questionnaire-based study identified eleven significant factors contributing to drug-induced states of consciousness: experience of unity; spiritual experience; blissful state; insightfulness; dis-embodiment; impaired control and cognition; anxiety; complex imagery; elementary imagery; audio-visual synesthesia; and changed meaning of percepts.

Altered states of consciousness might have been employed by humans as early as 30,000 years ago. Mindaltering plants and/or excessive dancing were used to attain an ecstatic or mystic state. Examples of early religious use of altered states of consciousness are the rites of Dionysos and the Eleusinian Mysteries, as well as Yoga and Meditation. Followers of various shamanic traditions "enter altered states of consciousness in order to serve their community." Meditation in its various forms is being rediscovered by modern psychology because of its therapeutic potential and its ability to "enable the activity of the mind to settle down". In Psychotherapy techniques like hypnosis, meditation, support psychological processes.

Due to the behaviourist paradigm in psychology altered states of consciousness were dismissed as a field of scientific inquiry during the early 20th century. The social change of the turbulent 1960 s has decisively led to a change of the scientific perspective to the point that introspection as a scientific method and ASCs as valid realms of experience became more widely accepted. Foundations for the research have been laid out by various scientists 
such as Abraham Maslow, Walter N. Pahnke, Stanislav Grof and Charles Tart. They focused on seemingly beneficial aspects of ASCs such as their potential to "promote creativity or treat addiction". In the midst of the rise of new-age subculture Stanislav Grof and others formed the new field of transpersonal psychology, which emphasized "the importance of individual human experience, validity of mystical and spiritual experience, interconnectedness of self with others and the world and potential of self-transformation".

I recommend the papar "States of consciousness in esoteric practice," by Petar Vujičin, focused on a comparative presentation of two esoteric practices: Upanishads known under term Vedanta (Hinduism) and esoteric Christianity by a contemporary Cypriot mystic Daskalos (teacher). The striking correspondences between these two traditions have been found, from the macrocosm, the structure of the Universe, to the microcosm, the structure of human selfhood, human bodies, human states of consciousness, and precise techniques for expansion of higher states of consciousness.

Also, a brief comment is made on the psychic phenomena in the esoteric tradition, as well as on the research of these phenomena carried out by Western scientists. Finally, it is maintained that implementation of esoteric disciplines and codes of behaviour in the many aspects of human life, personal and social, is the most efficient way for eliminating all evils (wars, crimes, poverty, illnesses, and diseases, unhappiness), having beset humanity in its long history.

\section{Additional recommended references:}

- https://en.wikipedia.org/wiki/Altered state of consciousness (Wikipedia), and refs. therein

- http://www.dejanrakovicfund.org/file/States_of_consciousness in_esoteric_practice.pdf

(P. Vujičin, "States of consciousness in esoteric practice," in: D. Raković, Dj. Koruga, editors, Consciousness:

Scientific Challenge of the 21st Century, ECPD, Belgrade, 1995, pp. 89-136)

\section{B.2. Holonomic Brain Theory [Adopted from Wikipedia]}

Holonomic brain theory is a branch of neuroscience investigating the idea that human consciousness is formed by quantum effects in or between brain cells. This is opposed by traditional neuroscience, which investigates the brain's behavior by looking at patterns of neurons and the surrounding chemistry, and which assumes that any quantum effects will not be significant at this scale. The specific theory of quantum consciousness was developed by neuroscientist Karl Pribram initially in collaboration with physicist David Bohm. It describes human cognition by modeling the brain as a holographic storage network. Pribram suggests these processes involve electric oscillations in the brain's fine-fibered dendritic webs, which are different from the more commonly known action potentials involving axons and synapses. Gabor, Pribram and others noted the similarities between these brain processes and the storage of information in a hologram, which can also be analyzed with a Fourier transform. In a hologram, any part of the hologram with sufficient size contains the whole of the stored information. In this theory, a piece of a long-term memory is similarly distributed over a dendritic arbor so that each part of the dendritic network contains all the information stored over the entire network. This model allows for important aspects of human consciousness, including the fast associative memory that allows for connections between different pieces of stored information and the non-locality of memory storage (a specific memory is not stored in a specific location, certain cluster of neurons).

While Pribram originally developed the holonomic brain theory as an analogy for certain brain processes, several later papers have proposed that the similarity between hologram and certain brain functions is more than just metaphorical, but actually structural. Several studies have shown that the same series of operations used in holographic memory models are performed in certain processes concerning temporal memory and optomotor responses. Other studies have demonstrated the possibility that biophoton emission (biological electrical signals that are converted to weak electromagnetic waves in the visible range) may be a necessary condition for the electric activity in the brain to store holographic images. A holographic model can also account for other features of memory that more traditional models cannot. The Hopfield memory model has an early memory saturation point before which memory retrieval drastically slows and becomes unreliable. On the other hand, holographic memory 
models have much larger theoretical storage capacities. Holographic models can also demonstrate associative memory, store complex connections between different concepts, and resemble forgetting through "lossy storage."

I recommend the book Holographic Universe to all interested in various states of consciousness, with numerous well-documented mysterious phenomena in altered states of consciousness. In this book, Michael Talbot relies heavily on the works of famous quantum physicist David Bohm and neurophysiologist Karl Pribram, who point out that the Universe could be a giant quantum hologram, the type of mind construct at the boundary of a subject / object - by relativizing the question of whether objective reality exists or constantly fluctuates between implicate \& explicate order?

The book suggests that many well documented mysterious phenomena (synchronicity \& precognition, extrasensory perceptions \& out-of-body experiences, psychokinesis \& teleportations, miraculous healing \& mystical religious experiences...) - could be explained within the (still controversial) holographic paradigm. The reading and presentation of this book brought me great personal satisfaction, for my own research of quantum basis of consciousness \& holistic psychosomatics and their implications (with numerous empirical / rational assertions, I recognized by reading a book).

Additional recommended references:

- https://en.wikipedia.org/wiki/Holonomic brain theory (Wikipedia), and refs. therein

- https://www.amazon.com/Holographic-Universe-Revolutionary-Theory-Reality/dp/0062014102

(M. Talbot, The Holographic Universe, HarperCollins, New York, 1991)

\section{B.3. Holography [Adopted from Wikipedia]}

Holography is the science and practice of making holograms. The holographic medium, produced by a holographic process (hologram) is usually unintelligible when viewed under diffuse ambient light. In its pure form, holography requires the use of laser light for illuminating the object and for viewing the finished hologram. Holography may be better understood via an examination of its difference from ordinary photography. When a photograph is cut in half, each piece shows half of the scene. When a hologram is cut in half, the whole scene can still be seen in each piece - because, whereas each point in a photograph only represents light scattered from a single point in the scene, each point on a holographic recording includes information about light scattered from every point in the scene.

Mathematical model [Adopted from Wikipedia]. A single-frequency light wave can be modeled by a complex number, $\boldsymbol{U}$, which represents the electric or magnetic field of the light wave. The amplitude and phase of the light are represented by the absolute value and angle of the complex number. The object and reference waves at any point in the holographic system are given by $\boldsymbol{U}_{O}$ and $\boldsymbol{U}_{R}$. The combined beam is given by $\boldsymbol{U}_{O}+\boldsymbol{U}_{R}$. If a photographic plate is exposed to the two beams and then developed, its transmittance $\boldsymbol{T}$ is proportional to the light energy proportional (with constant $k$ ) to the square of magnitude of the combined beams:

$$
T=k\left|U_{O}+U_{R}\right|^{2}=k U_{O} U_{R}^{*}+k\left|U_{R}\right|^{2}+k\left|U_{O}\right|^{2}+k U_{O}^{*} U_{R}
$$

When the developed plate is illuminated by the reference beam, the light transmitted through the plate, $\mathbf{U}_{H}$, is equal to the transmittance, $T$, multiplied by the reference beam amplitude, $\boldsymbol{U}_{R}$, giving

$$
U_{H}=T U_{R}=k U_{O}\left|U_{R}\right|^{2}+k\left|U_{R}\right|^{2} U_{R}+k\left|U_{O}\right|^{2} U_{R}+k U_{O}^{*} U_{R}^{2}
$$

It can be seen that $\boldsymbol{U}_{H}$ has four terms. The first term is proportional to $\boldsymbol{U}_{O}$, and is the reconstructed object beam, which enables a viewer to 'see' the original object even when it is no longer present in the field of view. The second and third beams are modified versions of the reference beam. The fourth term is the "conjugate object beam", and has the reverse curvature to the object beam itself and forms a real image of the object in the space beyond the holographic plate. When the reference and object beams are incident on the holographic recording 
medium at significantly different angles, the virtual, real, and reference wavefronts all emerge at different angles, enabling the reconstructed object to be seen clearly.

The Hungarian-British physicist Dennis Gabor was awarded the Nobel Prize in Physics in 1971 "for his invention and development of the holographic method". The technique as originally invented is still used in electron microscopy (known as electron holography). The development of the laser enabled the first practical optical holograms that recorded 3D objects to be made in 1962 by Yuri Denisyuk in the Soviet Union and by Emmett Leith and Juris Upatnieks at the University of Michigan, USA. Several types of holograms can be made. Transmission holograms are viewed by shining laser light through them and looking at the reconstructed image from the side of the hologram opposite the source. A later refinement, the "rainbow transmission" hologram, commonly used for security and authentication, allows more convenient illumination by white light rather than by lasers. Reflection holograms can also be viewed using a white-light illumination source on the same side of the hologram as the viewer and is the type of hologram normally seen in holographic displays; they are also capable of multicolour-image reproduction.

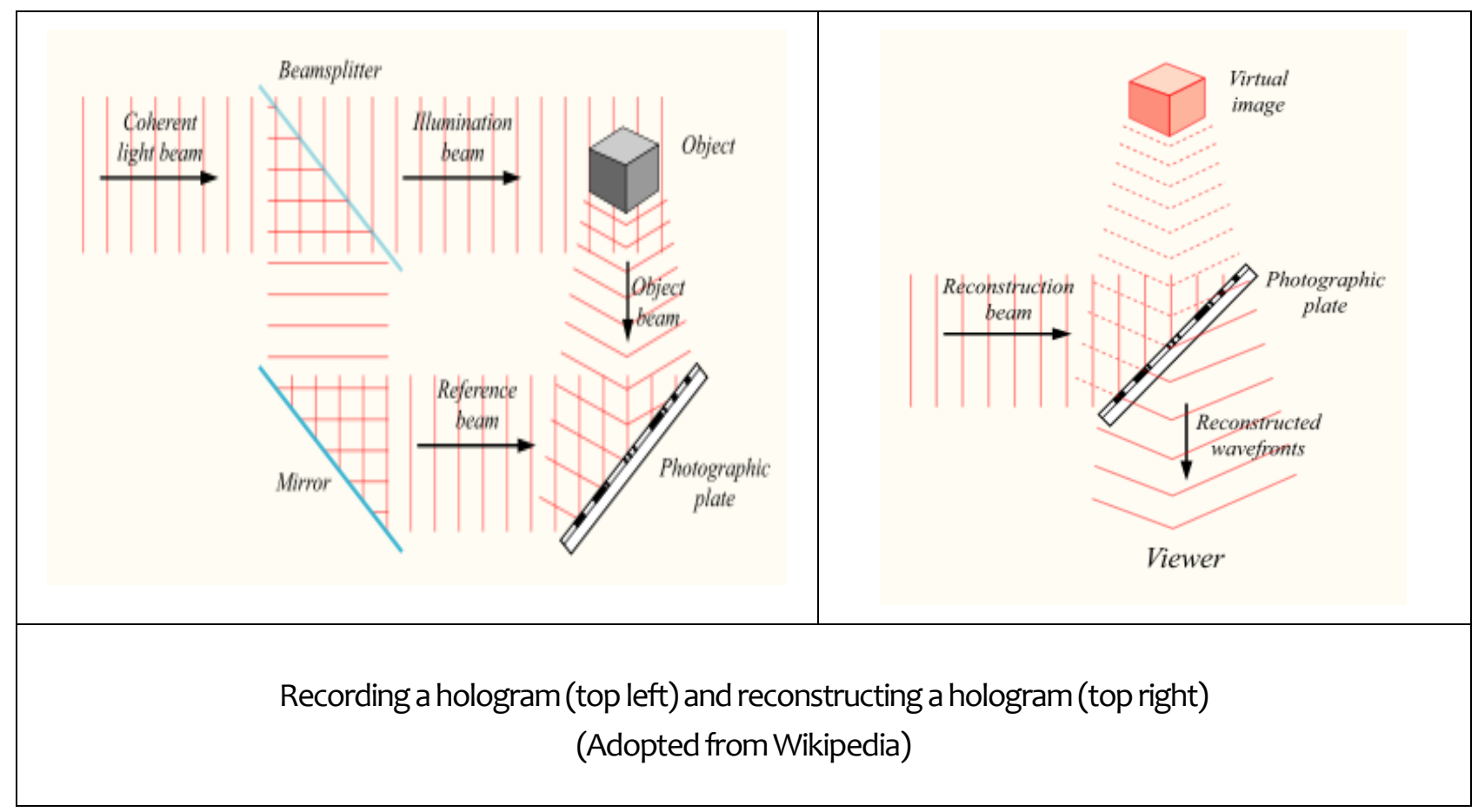

Additional recommended references:

- https://en.wikipedia.org/wiki/Holography (Wikipedia), and refs. therein

- https://www.amazon.com/Biological-Quantum-Computing-Human-Vision/dp/1615207856

(M. Peruš, C. K. Loo, Biological and Quantum Computing for Human Vision: Holonomic Models and Applications, Hershey, Medical Information Science Reference, 2011)

\section{B.4. Quantum Mechanics [Adopted from Wikipedia]}

Quantum mechanics (QM), including quantum field theory, is a fundamental theory in physics which describes nature at the smallest scales of energy levels of atoms and subatomic particles. Classical physics, the physics existing before QM, describes nature at ordinary (macroscopic) scale. Most theories in classical physics can be derived from QM as an approximation valid at large (macroscopic) scale. QM differs from classical physics in that energy, momentum, angular momentum and other quantities of a bound system are restricted to discrete values (quantization); objects have characteristics of both particles and waves (wave-particle duality); and there are limits to the precision with which quantities can be measured (uncertainty principle).

QM gradually aroses from theories to explain observations which could not be reconciled with classical physics, such as Max Planck's solution in 1900 to the black-body radiation problem, the correspondence between energy and frequency in Albert Einstein's 1905 paper which explained the photoelectric effect. Early quantum theory was profoundly re-conceived in the mid-1920s by Erwin Schrödinger, Werner Heisenberg, Max Born and others, when re- 
formulated in various specially developed mathematical formalisms. Applications of quantum theory include quantum chemistry, quantum optics, quantum computing, superconducting magnets, light-emitting diodes, laser, transistor, semiconductors such as microprocessor, medical and research imaging, like magnetic resonance imaging and electron microscopy. Explanations for many biological and physical phenomena are rooted in the nature of the chemical bond, most notably the macro-molecule DNA.

In the formalism of QM, the state of a system at a given time is described by a complex wave function, also referred to as state vector in a complex vector space. This abstract mathematical object allows for the calculation of probabilities of outcomes of concrete experiments. Contrary to classical mechanics, one can never make simultaneous predictions of conjugate variables, such as position and momentum, to arbitrary precision. The possible results of a measurement are the eigenvalues of the operator representing the observable - which explains the choice of Hermitian operators, for which all the eigenvalues are real. The probability distribution of an observable in a given state can be found by computing the spectral decomposition of the corresponding operator. Heisenberg's uncertainty principle is represented by the statement that the operators corresponding to certain observables do not commute.

In his treatise The Mathematical Foundations of Quantum Mechanicsin in 1932, John von Neumann deeply analyzed the so-called measurement problem, and concluded that the entire physical universe could be made subject to the Schrödinger equation (the universal wave function), and described how measurement could cause a collapse of the wave function (the projection postulate) (originally in the von Neumann's "chain": quantum object + apparatus + observer, but later on necessity of self-consciousness has been usually seen as non-physical solution of the problem). Later on, quantum decoherence framework developed in 1980 s for open quantum systems, has been used by Wojciech Zurek to provide an explanation for apparent wave-function collapse, as the quantum nature of the system "leaks" into the environment. A total superposition of the global or universal wavefunction still exists (and remains coherent at the global level), but its ultimate fate remains an interpretational issue. Specifically, quantum decoherence does not attempt to explain the measurement problem, but provides environment-induced superselection rules for transition of the system from quantum-coherent to classically reduced states.

Since its inception, many counter-intuitive aspects and results of QM have provoked strong philosophical debates and many interpretations. Even fundamental issues, such as Max Born's basic rules concerning probability amplitudes and probability distributions, took decades to be appreciated by society and many leading scientists. Richard Feynman once said, "I think I can safely say that nobody understands QM." According to Steven Weinberg, "There is now in my opinion no entirely satisfactory interpretation of QM." Although QM has held up to rigorous and extremely precise tests in an extraordinarily broad range of experiments (not one prediction from QM is found to be contradicted by experiments), there exist a number of contending schools of thought over interpretation of quantum mechanics. These views on interpretation differ on such fundamental questions as whether $Q M$ is deterministic or random, which elements of QM can be considered "real", and what is the nature of measurement, among other matters. Despite nearly a century of debate and experiment, no consensus has been reached amongst physicists and philosophers of physics concerning which interpretation best "represents" reality.

Copenhagen interpretation - due largely to Niels Bohr and Werner Heisenberg - remains most widely accepted amongst physicists, some 75 years after its enunciation. According to this interpretation, the probabilistic nature of QM is not a temporary feature which will eventually be replaced by a deterministic theory, but instead must be considered a final renunciation of the classical idea of "causality." It is also believed therein that any well-defined application of the QM formalism must always make reference to the experimental arrangement, due to the conjugate nature of evidence obtained under different experimental situations.

The probabilistic nature of QM thus stems from the act of measurement, one of the most difficult aspects of quantum systems to understand. It was the central topic in the famous Bohr-Einstein debates, which provide a vibrant critique of the Copenhagen Interpretation from an epistemological point of view. Einstein considered particular proposal for hidden variables to obviate the acausality and non-locality of quantum mechanical measurement. In arguing for his views, he produced a series of objections, including the most famous Einstein-Podolsky-Rosen (EPR) paradox.

John Bell showed that this EPR paradox led to experimentally testable differences between QM and theories that rely on added hidden variables. By the early 1980s, Alain Aspect's and other experiments had shown that Bell' inequalities were indeed violated in practice - so that there were in fact correlations of the kind suggested by QM. At first these just seemed like isolated esoteric effects, but by the mid-1990s, they were being codified in the field of quantum information theory, and led to constructions with names such as quantum cryptography and quantum 
teleportation. Entanglement, as demonstrated in Bell-type experiments, does not, however, violate causality, since no transfer of information happens.

Everett many-worlds interpretation, formulated in 1956, holds that all possibilities described by quantum theory simultaneously occur in a multiverse composed of mostly independent parallel universes. This is not accomplished by introducing some "new axiom" to QM, but on the contrary, by removing the axiom of the collapse of the wave packet. All of the possible consistent states of the measured system and the measuring apparatus (including the observer) are present in a real physical quantum entangled state. While the multiverse is deterministic, we perceive non-deterministic behavior governed by probabilities, because we can only observe the universe that we, as observers, inhabit. Everett's interpretation is fully consistent with Bell's inequalities and makes them intuitively understandable. However, according to the theory of quantum decoherence, these "parallel universes" are not accessible to us.

De Broglie-Bohm theory (also known as the pilot wave theory) is a theory of QM by Louis de Broglie and extended later by David Bohm to include measurements. Particles, which always have positions, are guided by the wavefunction. The wavefunction evolves according to the Schrödinger wave equation, and the wavefunction never collapses. The theory takes place in a single space-time, is non-local, and is deterministic. The simultaneous determination of a particle's position and velocity is subject to the usual uncertainty principle constraint. The theory is considered to be a hidden-variable theory, and by embracing non-locality it satisfies Bell's inequality. The measurement problem is resolved, since the particles have definite positions at all times. Collapse is explained as phenomenological.

Objective collapse theories differ from the Copenhagen interpretation by regarding both the wave function and the process of collapse as ontologically objective (meaning these exist and occur independent of the observer). In objective theories, collapse occurs either randomly ("spontaneous localization") or when some physical threshold is reached, with observers having no special role. Thus, objective-collapse theories are realistic, indeterministic, nohidden-variables theories, requiring for an extension of QM. Examples include: Ghirardi-Rimini-Weber theory and Penrose interpretation.

In general, only closed composite quantum system $S+E$ (composed of interacting open quantum subsystems $S$ and $E$ ) is subject to Schrödinger law (with quantum entanglement or quantum correlations of the states of subsystems $S$ and $E$, as explicit on the r.h.s. in Eq. (I))

$$
|\mathrm{X}\rangle_{S+E} \equiv \hat{U}|\Phi\rangle_{S}|\Psi\rangle_{E}=\sum_{i} c_{i}\left|\Phi_{i}\right\rangle_{S}\left|\Psi_{i}\right\rangle_{E}
$$

$\widehat{U}(t)=\exp (-2 \pi i \hat{H} t / h)$ denotes the unitary time-evolution operator with Hamiltonian $\widehat{H}=\hat{H}_{S}+\hat{H}_{E}+\hat{H}_{\text {int }}$, and $\left|\Phi_{i}\right\rangle_{S}$ and $\left|\Psi_{i}\right\rangle_{E}$ constitute orthonormalized bases in the Hilbert state spaces of subsystems $S$ and $E$ (considered initially approximately isolated / closed), where interaction Hamiltonian $\hat{H}_{\text {int }}$ depends on observables of both subsystems $S$ and $E$. It should be pointed out that Schrödinger law does not hold true separately for neither $S$ nor $E$ as interacting open quantum subsystems, when two-stage quantum decoherence terminates (cf. Eqs. (I) \& (II), or Eq. (1) in Footnote 10) into classicaly-reduced states of finally decoupled subsystems $S$ and $E$, as explicit on the r.h.s. in Eq. (II)):

$$
|\Phi\rangle_{S}|\Psi\rangle_{E} \rightarrow|\mathrm{X}\rangle_{S+E} \equiv \sum_{i} c_{i}\left|\Phi_{i}\right\rangle_{S}\left|\Psi_{i}\right\rangle_{E} \stackrel{p_{j}=\left|c_{j}\right|^{2}}{\longrightarrow}\left|\Phi_{j}\right\rangle_{S}\left|\Psi_{j}\right\rangle_{E}
$$

On the other hand, when $\hat{H}_{\text {int }}$ can be reduced to the "external field", its potential term $\widehat{V}$ can be added to $\hat{H}_{S}$, providing new self-Hamiltonian of the subsystem $S$, dynamically decoupled from the observables of the environmental subsystem $E$, and then subsystem $S$ can be treated as the closed quantum system. This is the case, for instance, in most situations in quantum chemistry, with Schrödinger equation applied to the explored closed many-atomic quantum system with appropriate boundary conditions and adopted computational approximations (giving rise to stationary ground and excited electronic-vibrational energy eigenstates of all possible many-atomic isomers. It should be noted that Schrödinger equation cannot apply to nonstationary excitations and relaxations of the many-atomic quantum system, not only in-between different isomers but also 
within the same isomer - when quantum deexcitation / decoherence must apply to non-potential interaction of the open many-atomic quantum subsystem $S$ (non-describable fully by its self-Hamiltonian) with its quantum environment $E$ (generally field-related, including vacuum).

Consciousness causes collapse interpretation includes John Archibald Wheeler's participatory anthropic principle which says that consciousness plays some role in bringing the universe into existence. Originally, John von Neumann deeply analyzed the so-called measurement problem, and concluded that the "collapse" is ultimatively referred to self-consciousness of the observer, as the final element of the von Neumann's "chain" (quantum object + apparatus + observer). This point of view was prominently expanded on by Eugene Wigner, who argued that experimenter consciousness was critical for the collapse.

Quantum information theories have attracted growing support. They subdivide into two kinds: Information ontologies, such as J. A. Wheeler's "it from bit", being described as a revival of immaterialism; Interpretations where QM is said to describe an observer's knowledge of the world, with some similarity with Bohr's thinking, being described as similar to instrumentalism.

Additional recommended references:

- https://en.wikipedia.org/wiki/Quantum_mechanics (Wikipedia), and refs. therein

- http://www.dejanrakovicfund.org/knjige/2009-Integ-Biophys-Quant-Medic.pdf

(D. Raković, Integrative Biophysics, Quantum Medicine, and Quantum-Holographic Informatics: Psychosomatic-Cognitive Implications, IASC \& IEPSP, Belgrade, 2009, Apps. 6.1-6.9, and refs. therein)

- http://www.dejanrakovicfund.org/papers/2002-INFORMATICA.pdf

(D. Raković, M. Dugić, "A critical note on the role of the quantum mechanical 'collapse' in quantum modeling of consciousness", Informatica, vol. 26, no. 1(2002), pp. 85-90)

\section{B.5. Neural Networks [Adopted from Wikipedia]}

Neural networks were originally based on efforts to model information processing in biological systems (contrary to digital computers evolved from the von Neumann model, which operate via the execution of explicit instructions via access to memory by a number of processors).

Biological neural network (BNN) is composed of a group or groups of chemically connected or functionally associated neurons. A single neuron may be connected to many other neurons and the total number of neurons and connections in a network may be extensive (up to 40,000). Connections, called synapses, are usually formed from axons to dendrites, though dendrodendritic synapses and other connections are possible. Apart from the electrical signaling, there are other forms of signaling arising from neurotransmitter diffusion. Artificial neural network (ANN) is composed of artificial neurons or nodes. Thus a neural network is either a BNN made up of real biological neurons, or an ANN that changes its structure based on external or internal information that flows through the network, for solving artificial intelligence (AI) problems, in speech recognition, image analysis and adaptive control, in order to construct software agents (in computer and video games) or autonomous robots.

The connections of the biological neuron are modeled as weights. A positive weight reflects an excitatory connection, while negative values mean inhibitory connections. All inputs are modified by a weight and summed. Finally, an activation function controls the amplitude of the output. For example, an acceptable range of output is usually between 0 and 1 , or it could be -1 and 1 . Artificial neurons were first proposed in 1943 by Warren McCulloch, a neurophysiologist, and Walter Pitts, a logician, who first collaborated at the University of Chicago. One classical type of ANNs is the recurrent Hopfield network. The concept of a NN appears to have first been proposed by Alan Turing in his 1948 paper Intelligent Machinery. 


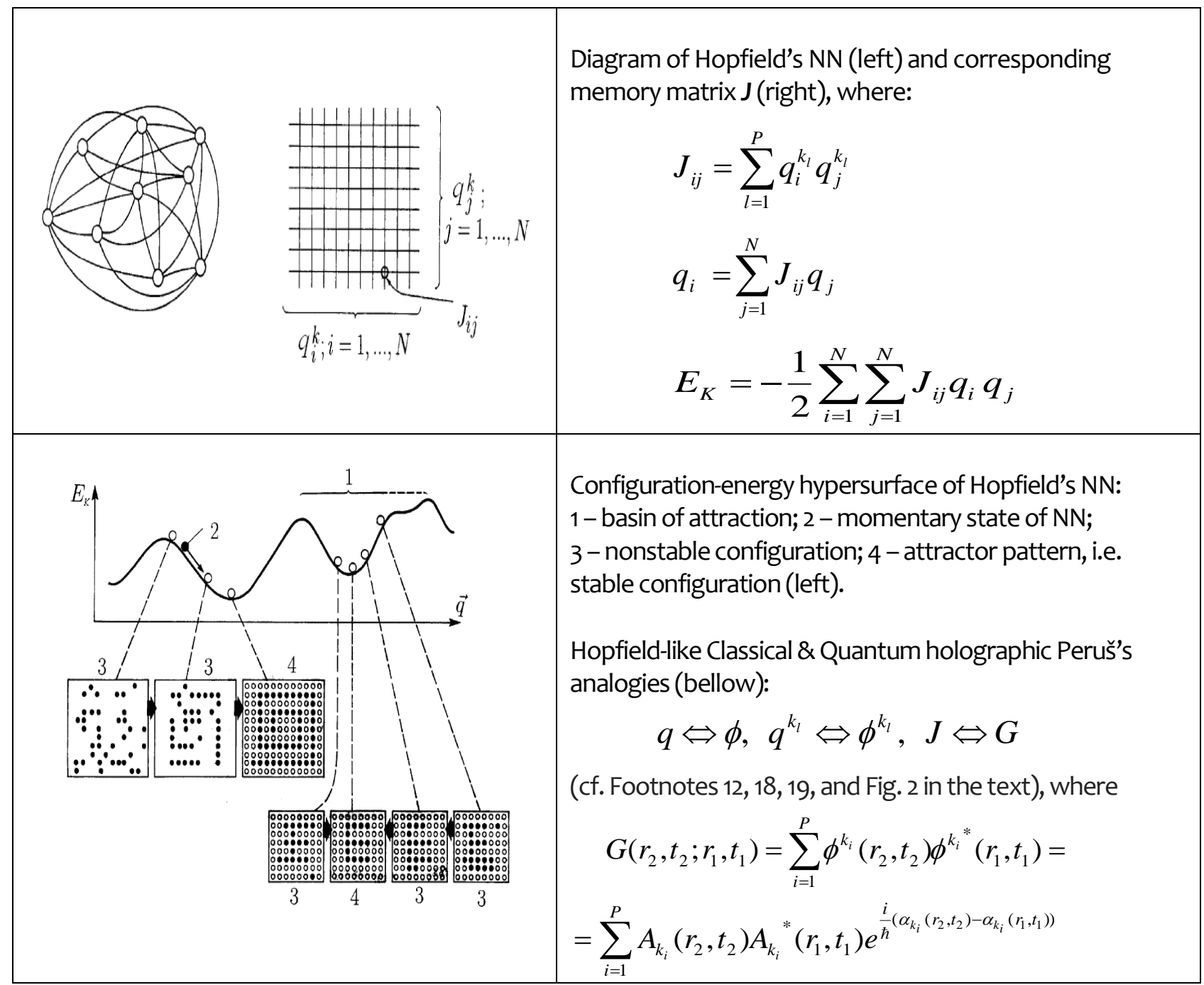

The utility of ANN models lies in the fact that they can be used to infer a function from observations and also to use it. Unsupervised ANNs can also be used to learn representations of the input that capture the salient characteristics of the input distribution, like in deep learning algorithms, which can implicitly learn the distribution function of the observed data. Learning in ANNs is particularly useful in applications where the complexity of the data or task makes the design of such functions by hand impractical.

Additional recommended references:

- https://en.wikipedia.org/wiki/Neural_network(Wikipedia), and refs. therein

- http://www.dejanrakovicfund.org//knjige/2009-Integ-Biophys-Quant-Medic.pdf

(D. Raković, Integrative Biophysics, Quantum Medicine, and Quantum-Holographic Informatics: Psychosomatic-Cognitive Implications, IASC \& IEPSP, Belgrade, 2009, Chs. 2, 4, 5, and refs. therein)

\section{B.6. Entropy In Thermodynamics \& Information Theory [Adopted from Wikipedia]}

The defining expression for entropy in the theory of statistical mechanics established by Ludwig Boltzmann and J. Willard Gibbs in 1870 s is: $S=-k_{B} \sum_{i} p_{i} \ln p_{i}$, where $p_{i}$ is the probability of the microstate i taken from an equilibrium ensemble, and $k_{B}$ is Boltzmann constant. If all the microstates are equiprobable (a microcanonical ensemble), statistical thermodynamic entropy reduces to form given by Boltzmann: $S=k_{B} \ln W$, where $W$ is the number of microstates that corresponds to macroscopic thermodynamic state, and depends on temperature. The logarithm in the thermodynamic definition is the natural logarithm. It can be shown that the Gibbs entropy formula reproduces all of the properties of the macroscopic classical thermodynamics of Rudolf Clausius. In quantum statistical mechanics, described by a density matrix $\rho$, the extension of classical Gibbs entropy is von Neumann entropy, $-\operatorname{Tr}(\rho \ln \rho)=-\langle\ln \rho\rangle$. 
The defining expression for entropy in the theory of information established by Claude Shannon in his article $A$ Mathematical Theory of Communication in 1948 is: $H=-\sum_{i} p_{i} \log _{2} p_{i}$, where $p_{i}$ is the probability of the message $m_{i}$ taken from the message space $M$. Information entropy is calculated using base 2 logarithms, when unit of the entropy is bit. Mathematically $\mathrm{H}$ may be seen as an average information, taken over the message space.

Despite the foregoing similarities, there is a difference between the two quantities. The information entropy $\mathrm{H}$ can be calculated for any probability distribution (if the "message" is taken to be that the event $i$ which had probability $p_{i}$ occurred, out of the space of the events possible), while the thermodynamic entropy Srefers to thermodynamic probabilities $p_{i}$ specifically. The difference is more theoretical than actual, however, because any probability distribution can be approximated arbitrarily closely by some thermodynamic system.

Shannon's entropy has been related by physicist Léon Brillouin to a concept sometimes called negentropy. In 1953, Brillouin derived a general equation stating that the changing of an information bit value requires at least $k T \ln (2)$ energy. This is the same quantity found by Rolf Landauer in 1961. In his book, he further explored this problem concluding that any cause of a bit value change (measurement, decision about a yes/no question, erasure, display, etc.) will require the same amount, $k T \ln (2)$, of energy. Consequently, acquiring information about a system's microstates is associated with an entropy production, while erasure yields entropy production only when the bit value is changing. Setting up a bit of information in a sub-system originally in thermal equilibrium results in a local entropy reduction. However, there is no violation of the second law of thermodynamics, according to Brillouin, since a reduction in any local system's thermodynamic entropy results in an increase in thermodynamic entropy elsewhere.

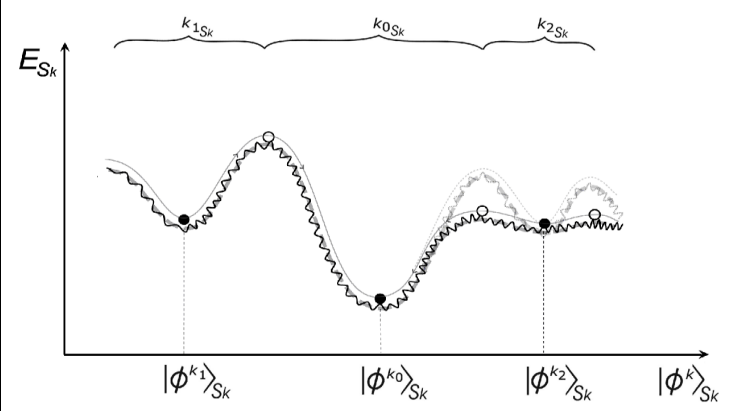

Schematic bioresonant balansing of unwilling side memory attractors in the energy-state hypersurface of various hierarchical levels of biological macroscopic open quantum system $S_{\mathrm{k}}$, which would otherwise cause development of psychosomatic diseases on individual level, and interpersonal fights on collective level.
By considering psychosomatic state in generally mixed quantum state of density matrix

$$
\widehat{\rho}_{S}=\sum_{i}\left|c_{k_{i}}\right|^{2}\left|\phi^{k_{i}}\right\rangle_{S}\left\langle\phi^{k_{i}}\left|\equiv \sum_{i} p_{k_{i}}\right| \phi^{k_{i}}\right\rangle_{S}\left\langle\phi^{k_{i}}\right|
$$

von Neumann's entropy $S=-k_{B} \operatorname{Tr}\left(\hat{\rho}_{S} \ln \hat{\rho}_{S}\right)$ is reduced to Shannon's entropy $S=-k_{B} \sum_{i} p_{k_{i}} \ln p_{k_{i}}$. Then within our $Q D Q H$ theoretical framework, entropy of pure-healthy state of psychosomatic system (with single $k_{0}$-th term in superposition of probability $\left.p_{k_{0}}=1\right)$ is plausably minimal ( $S_{k_{0}}=0$, as pure quantum state $\hat{\rho}_{S_{k v}}^{\left(k_{0}\right)}$ provides maximal possible information about quantum psychosomatic system), while entropy of disordered state of psychosomatic system (described by full above superposition) is higher ( $S_{\hat{\rho}}>0$, as mixed stochastic state $\hat{\rho}_{S_{k v}}^{(k)}$ provides uncomplete information about quantum psychosomatic system).

\section{Additional recommended references:}

- https://en.wikipedia.org/wiki/Entropy_in thermodynamics_and_information_theory (Wikipedia), and refs. therein

- $\quad$ http://www.dejanrakovicfund.org/knjige/2009-Integ-Biophys-Quant-Medic.pdf (D. Raković, Integrative Biophysics, Quantum Medicine, and Quantum-Holographic Informatics: Psychosomatic-Cognitive Implications, IASC \& IEPSP, Belgrade, 2009, App. 6.5, and refs. therein)

\section{B.7. Holographic Principle [Adopted from Wikipedia]}

Holographic principle is a tenet of string theories and a supposed property of quantum gravity that states that the description of a volume of space can be thought of as encoded on a lower-dimensional boundary to the region such as a light-like boundary like a gravitational horizon. First proposed by Gerard 't Hooft, it was given a precise string-theory interpretation by Leonard Susskind who combined his ideas with previous ones of ' $t$ Hooft and Charles 
Thorn (who observed in 1978 that string theory admits a lower-dimensional description in which gravity emerges from it in what would now be called a holographic way).

The prime example of holography is the AdS/CFT correspondence, sometimes called Maldacena duality or gravity/gauge duality. On one side are anti-de Sitter spaces (AdS) which are used in theories of quantum gravity, formulated in terms of string theory or M-theory. On the other side of the correspondence are conformal field theories (CFT) which are quantum field theories, including theories similar to the Yang-Mills theories that describe elementary particles. AdS/CFT duality represents a major advance in our understanding of string theory and quantum gravity.

The holographic principle was inspired by black hole thermodynamics, which conjectures that the maximal entropy in any region scales with the radius squared, and not cubed as might be expected. In the case of a black hole, the insight was that the informational content of all the objects that have fallen into the hole might be entirely contained in surface fluctuations of the event horizon. The holographic principle resolves the black hole information paradox within the framework of string theory.

In an article in the August 2003 issue of Scientific American titled "Information in the holographic universe", Bekenstein summarizes that "Thermodynamic entropy and Shannon entropy are conceptually equivalent: the number of arrangements that are counted by Boltzmann entropy reflects the amount of Shannon information one would need to implement any particular arrangement" of matter and energy. The only salient difference between the thermodynamic entropy of physics and Shannon's entropy of information is in the units of measure; the former is expressed in units of energy divided by temperature, the latter in essentially dimensionless "bits" of information. As the holographic principle states that the entropy of ordinary mass (not just black holes) is also proportional to surface area and not volume, Bekenstein conjectures that volume itself is illusory and the universe is really a hologram which is isomorphic to the information "inscribed" on the surface of its boundary.

Additional recommended references:

- https://en.wikipedia.org/wiki/Holographic_principle(Wikipedia), and refs. therein

- http://staff.ustc.edu.cn/ wzhaoz/c index files/main.files/blackhole.pdf

(L. Susskind, J. Lindesay, An Introduction to Black Holes, Information and the String Theory Revolution: The Holographic Universe, World Scientific, Singapore, 2005)

\section{B.8. String Theory [Adopted from Wikipedia]}

String theory is a theoretical framework in which the point-like particles are replaced by one-dimensional strings. It describes how these strings propagate through space and interact with each other. On distance scales larger than the string scale, a string looks like an ordinary particle, with its mass, charge, and other properties determined by the vibrational state of the string. In string theory, one of the many vibrational states of the string corresponds to the graviton, a quantum mechanical particle that carries gravitational force. Thus string theory is a theory of quantum gravity.

String theory has been applied to a variety of problems in black hole physics, early universe cosmology, nuclear physics, and condensed matter physics, and it has stimulated a number of major developments in pure mathematics. Because string theory potentially provides a unified description of gravity and particle physics, it is a candidate for a theory of everything (TOE), which describes all fundamental forces and forms of matter. Despite much work on these problems, it is not known to what extent string theory describes the real world or how much freedom the theory allows in the choice of its details.

String theory was first studied in the late 1960s. Subsequently, it was realized that the very properties that made string theory unsuitable as a theory of nuclear physics made it a promising candidate for a quantum theory of gravity. The earliest version of string theory, bosonic string theory, incorporated only the class of particles known as bosons. It later developed into superstring theory, which posits supersymmetry between bosons and the class of particles known as fermions. In the mid-1990s it was realized that there is only one limiting case of superstring theories in eleven dimensions known as M-theory. In late 1997, an important relationship called the AdS/CFT correspondence related string theory to a quantum field theory. 


Interaction in the quantum world: worldlines of point
like particles (left) or a worldsheet swept up by closed strings in string
theory (right).
(Adopted from Wikipedia)

Additional recommended references:

- https://en.wikipedia.org/wiki/String_theory (Wikipedia), and refs. therein

- http://staff.ustc.edu.cn/ wzhao /c_index_files/main.files/blackhole.pdf

(L. Susskind, J. Lindesay, An Introduction to Black Holes, Information and the String Theory Revolution: The Holographic Universe, World Scientific, Singapore, 2005)

\section{B.9. Quantum Gravity [Adopted from Wikipedia]}

Quantum gravity (QG) is a field of theoretical physics that seeks to describe gravity according to the principles of quantum mechanics, and where quantum effects cannot be ignored, such as near compact astrophysical objects where the effects of gravity are strong. The current understanding of gravity is based on Albert Einstein's general theory of relativity, formulated within the framework of classical physics. On the other hand, the other three fundamental forces of physics are described within the framework of quantum mechanics and quantum field theory, radically different formalisms for describing physical phenomena. It is sometimes argued that a quantum mechanical description of gravity is necessary on the grounds that one cannot consistently couple a classical system to a quantum one.

While a quantum theory of gravity may be needed to reconcile general relativity with the principles of quantum mechanics, difficulties arise when applying the usual prescriptions of quantum field theory to the force of gravity via graviton bosons. The problem is that the theory one gets in this way is not renormalizable (it predicts infinite values for some observable properties such as the mass of particles) and therefore cannot be used to make meaningful physical predictions. As a result, theorists have taken up more radical approaches to the problem of QG, the most popular approaches being string theory and loop quantum gravity. So string theory tries to unify gravity with other fundamental forces, aiming to form a quantum field theory of gravity unified with a grand unified theory, so called theory of everything (TOE), while loop quantum gravity makes an effort only to quantize the gravitational field while kept separate from the other forces.

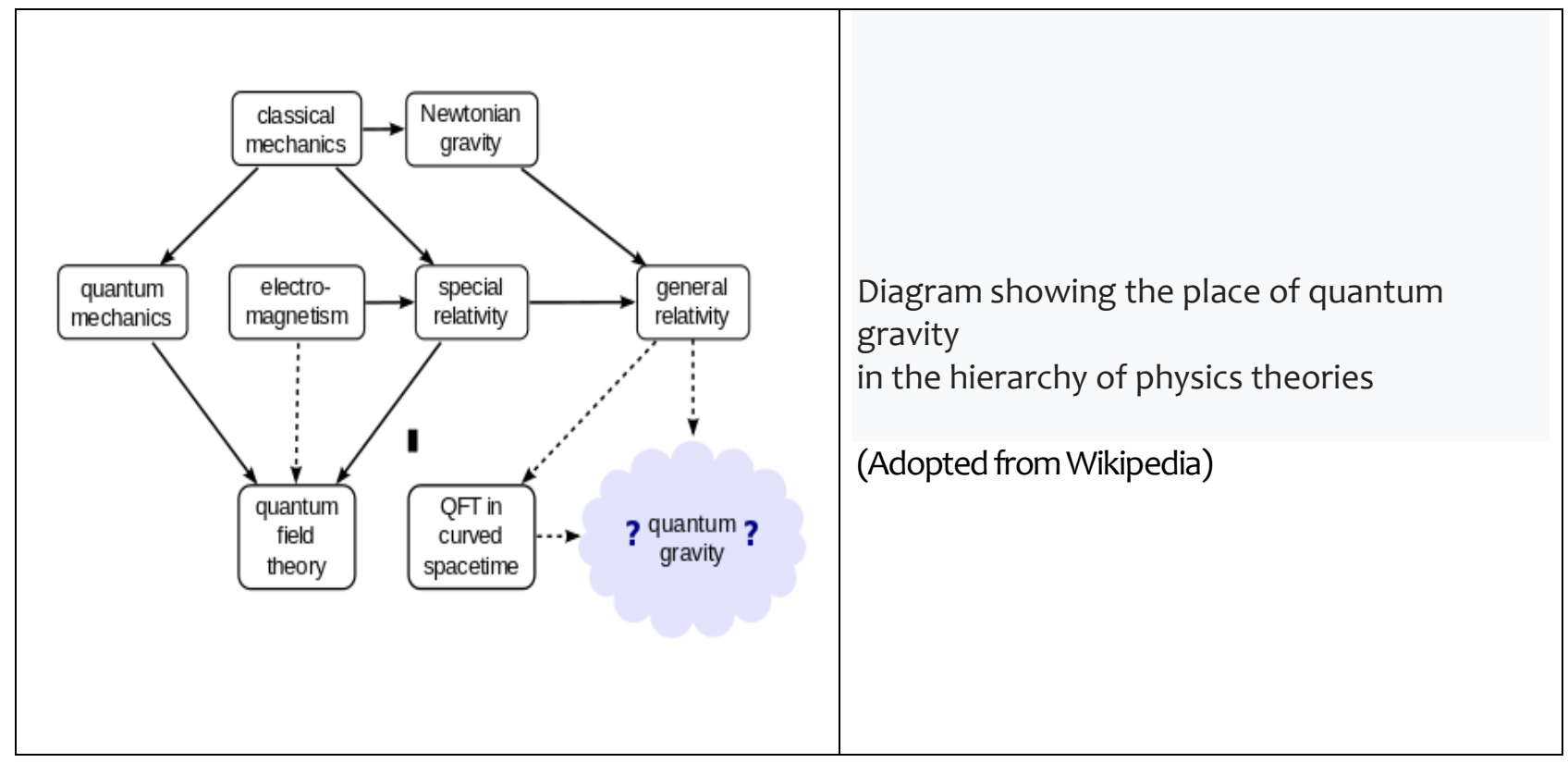

Additional recommended references:

- $\quad$ https://en.wikipedia.org/wiki/Quantum_gravity (Wikipedia), and refs. therein

- https://www.amazon.com/Reality-Not-What-Seems-Journey/dp/0735213933?ref_=fsclp_pl_dp 3 


\section{B.10. Quantum Field Theory [Adopted from Wikipedia]}

Quantum field theory (QFT) is a theoretical framework that combines classical field theory, special relativity, and quantum mechanics and is used to construct physical models of subatomic particles (in particle physics) and quasiparticles (in condensed matter physics).

QFT treats particles as excited states (also called quanta) of their underlying fields, which are - in a sense more fundamental than the basic particles. Interactions between particles are described by interaction terms in the Lagrangian involving their corresponding fields. Each interaction can be visually represented by Feynman diagrams, which are formal computational tools, in the process of relativistic perturbation theory.

As a successful theoretical framework today, QFT emerged from the work of generations of theoretical physicists spanning much of the 20th century. Its development began in the 1920s with the description of interactions between light and electrons, culminating in first QFT - quantum electrodynamics. A major theoretical obstacle soon followed with the appearance and persistence of various infinities in perturbative calculations, a problem only resolved in 1950 s with invention of the renormalization procedure. A second major barrier came with QFT's apparent inability to describe the weak and strong interactions, to the point where some theorists called for the abandonment of the field theoretic approach. The development of gauge theory and the completion of the Standard Model in the 1970 s led to a renaissance of QFT.

Additional recommended references:

- $\quad$ https://en.wikipedia.org/wiki/Quantum field theory (Wikipedia), and refs. therein

- https://www.amazon.com/Quantum-Field-Theory-Gifted-Amateur/dp/019969933X

(S. Blundell, T. Lancaster, Quantum Field Theory for the Gifted Amateur, Oxford Univ. Press, UK, 2014)

Acknowledgements - This paper is partly financed by the Serbian Ministry of Education, Science and Technological Development, Project No. 178027 (coordinated by Life Activities Advancement Center (LAAC), Belgrade).

\section{References}

[1] http://www.atpweb.org; official website of The Association for Transpersonal Psychology (ATP), concerned from early 1970 s with the study of humanity's highest potential, and with the recognition, understanding, and realization of unitive, spiritual, and transcendent states of consciousness (making transpersonal psychology the fourth force in psychology, alongside with psychoanalysis, behaviorism, and humanistic psychology, after one of its founders, A. Maslow).

[2] https://www.imconsortium.org; official website of the Consortium of Academic Medical Centers for Integrative Medicine, founded by the end of 1990s, which includes over 70 highly esteemed US academic health centers and affiliate institutions, in order to transform medicine and healthcare through rigorous scientific studies, new models of clinical care, and innovative educational programs that integrate biomedicine, the complexity of human beings, the intrinsic nature of healing, and the rich diversity of therapeutic systems.

[3] D. Raković, S. Arandjelović, M. Mićović, eds., Proc. Symp. Quantum-Informational Medicine QIM 2011: AcupunctureBased \& Consciousness-Based Holistic Approaches \& Techniques, QUANTTES \& HF \& DRF, Belgrade, 2011, and refs. therein.

[4] S. Hameroff et al, eds., Toward a Science of Consciousness, Series of Tucson Conferences, 1994-

[5] D. Raković, Dj. Koruga, eds., Consciousness: Scientific Challenge of the 21st Century, ECPD, Belgrade, 1995.

[6] J.von Neumann, Mathematical Foundations of Quantum Mechanics, Princeton Univ. Press, Princeton, 1955.

[7] S. Hameroff, R. Penrose, "Consciousness in the universe, A review of the 'Orch OR' theory," Phys Life Rev, vol. 11, pp. 39-78, 2014.

[8] R. Penrose, The Emperor's New Mind, Oxford Univ Press, New York, 1989.

[9] D. Raković, M. Dugić, M. M. Ćirković, "Macroscopic quantum effects in biophysics and consciousness," NeuroQuantology, vol. 2(4), pp. 237-262, 2004, and refs. therein.

[10] D. Raković, Integrative Biophysics, Quantum Medicine, and Quantum-Holographic Informatics: PsychosomaticCognitive Implications, IASC \& IEPSP, Belgrade, 2009; and refs therein. 
[11] D. Raković, "Tesla's legacy for new millennium: Path to quantum-holographic primal source," Int. Congress Nikola Tesla 2017: Disruptive Innovation, Belgrade, 02 July 2017, Invited lecture, and refs. therein; Preprint: http://www.dejanrakovicfund.org/radovi/2017_TESLA_Ple.pdf

[12] D. Raković, "On extended quantum-holographic framework for control of macro-quantum correlations of individual and collective consciousness," in: B. Reljin, S. Stanković, eds, Proc. 14th NEUREL, IEEE Serbia \& Montenegro Section, Belgrade, 2018, paper S2.5, and refs. therein.

[13] H. Stapp, Mind, Matter, and Quantum Mechanics, Springer, New York \& Berlin, 1993.

[14] M. Tegmark, "Importance of quantum decoherence in brain processes," Phys. Rev. E, vol. 61, pp. 4194-4206, 2000.

[15] K. S. Thorne, Black Holes and Time Warps: Einstein's Outrageous Legacy, Picador, London, 1994.

[16] J. Maldacena, L. Susskind, "Cool horizons for entangled black holes," Fortschr. Phys., vol. 61(9), pp. 781-811, 2013.

[17] P. J. E. Peebles, B. Ratra, "The cosmological constant and dark energy," Rev Mod Phys, vol. 75(2), pp. 559-606, 2003.

[18] J. A. Wheeler, K. W. Ford, Geons, Black Holes, and Quantum Foam: A Life in Physics, Norton, New York, 2000.

[19] M. Eliade, H. S. Wiesner, I. P. Couliano, The Eliade Guide to World Religions, Harper, San Francisco, 1991.

[20] L. McTaggart, The Intention Experiment: Using Your Thoughts to Change Your Life and the World, Free Press, New York, 2007.

[21] M. Talbot, The Holographic Universe, HarperCollins, New York, 1991.

[22] K. Wilber, The Atman Project, Quest, Wheaton, 1980.

[23] P.Vujičin, "States of consciousness in esoteric practice," in: Ref. 5, pp. 89-136.

[24] R. G. Jahn, "The persistent paradox of psychic phenomena: an engineering perspective," Proc. IEEE, vol. 70, pp. $136-170,1982$.

[25] J. Vlahos, Orthodox Psychotherapy: The Holy Fathers Science, Missionary School of St. Alexander Nevskiy Church, Belgrade, 1998; Serbian translation from Greek (1994).

[26] L. Dossey, Healing Words: The Power of Prayer and The Practice of Medicine, Harper, New York, 1993.

[27] D. Raković, "On quantum-holographic bases and frontiers of integrative medicine and transpersonal psychology: Psychosomatic, epistemological, and spiritual implications," in: G. Brekhman, M. Sovilj, D. Raković., eds., Proc. 1st Int. Congress on Psychological Trauma: Prenatal, Perinatal \& Postnatal Aspects (PTPPPA 2015), LAAC / IEPSP, Belgrade, 2015, pp. 59-72, and refs. therein.

[28] S. P. Sit'ko, "The realization of genome in the notions of Physics of the Alive," MD Medical Data, vol. 4(2), pp. 207215, 2012, and refs. therein.

[29] J.-M. Lourtioz, H. Benisty, V. Berger, J.-M. Gerard, D. Maystre, A. Tchelnokov, Photonic Crystals, 2nd ed., Springer, Berlin, 2008.

[30] D. Raković, "Quantum-informational bases and frontiers of psychosomatic integrative medicine," in: B. Reljin, S. Stanković, eds., Proc. 12th NEUREL, IEEE Serbia \& Montenegro Section, Belgrade, 2014, pp. 105-110 (similar Plenary lecture presented at: 7th Europ. Congress for Integrative Medicine (ECIM 2014), Belgrade, 10-11 October 2014); and refs. therein.

[31] W. R. Adey, "Tissue interactions with nonionizing electromagnetic fields," Physiol. Rev., vol. 61, pp. 435-514, 1981.

[32] M. Jibu, K. H. Pribram, K. Yasue, "From conscious experience to memory storage and retrieval: The role of quantum brain dynamics and boson condensation of evanescent photons," Intern. J. Mod. Phys., vol. 10, pp. 1735$1754,1996$.

[33] L. Susskind, J. Lindesay, An Introduction to Black Holes, Information and the String Theory Revolution: The Holographic Universe, World Scientific, Singapore, 2005.

[34] J. D. Bekenstein, "Information in the holographic universe," Scientific American, vol. 289(2), pp. 58-65, 2003.

[35] R. J. Callahan, J. Callahan, Thought Field Therapy and Trauma: Treatment and Theory, Indian Wells, 1996.

[36] M. Rakočević," "The universal consciousness and the universal code," in: Ref. 5, pp. 264-282.

[37] Y. Zhang, ECIWO Biology and Medicine: A New Theory of Conquering Cancer and Completely New Acupuncture Therapy, Neimenggu People Press, Beijing, 1987.

[38] P. P. Gariaev, M. J. Friedman, E. A. Leonova-Gariaeva, "Principles of linguistic-wave genetics, “ DNA Decipher J., vol. 1(1), pp. 11-24, 2011, and refs. therein.

[39] M. Newton, Journey of Souls, Llewellyn, Woodbury, 1994.

[40] D. Raković, M. Dugić, J. Jeknić-Dugić, M. Plavšić, S. Jaćimovski, J. Šetrajčić, "On macroscopic quantum phenomena in biomolecules and cells: From Levinthal to Hopfield," BioMed. Res. Int., vol. 2014, Article ID 580491, 9 pages, 2014, and refs. therein.

[41] M. Peruš, "Neuro-quantum parallelism in mind-brain and computers," Informatica, vol. 20, pp. 173-183, 1996. 
[42] Swami Prabhavananda, Ch. Isherwood (tr.), The Yoga Sutras of Patanjali. How to Know God, New American Library, New York, 1969.

[43] N. Berdyaev, Philosophy of Freedom, Logos Ant, Beograd, 1996; Serbian translation from Russian (1911).

[44] C. Castaneda, A Separate Reality: Further Conversations with Don Juan, Simon \& Schuster, New York, 1971.

[45] T. Rey, K. Richman, "The somatics of syncretism: Tying body and soul in Haitian religion," Studies in ReligionSciences Religieuses, vol. 3, pp. 279-403, 2010.

[46] D. Raković, "On bioresonant pattern-qua-attractors and healing boundary conditions within quantumholographic psychosomatics," in: B. Reljin, S. Stanković, eds., Proc. 13th NEUREL, IEEE Serbia \& Montenegro Section, Belgrade, 2016, pp. 9-13, and refs. therein.

[47] D. Raković, "On quantum-holographic and trans-generational implications for child developement," in: J. R. G. Turner, T. G. N. Turner, O. Gouni, eds, Prenatal Psychology 100 Years: A Jorney in Decoding How Our Prenatal Experience Shapes Who We Become!, A publication of The International Journal of Prenatal \& Life Sciences, Cosmoanelixis, Athens, 2018, Ch. 7, pp. 283-310, and refs. therein.

[48] R. Hecht-Nielsen, Neurocomputing, Addison-Wesley, New York, 1990.

[49] D. Panajotović, Buddhism: Guide to Theravada Buddhism, Udruženje srpskih izdavača, Belgrade, 2011, in Serbian.

[50] D. C. Dennett, Consciousness Explained, Little Brown, Boston, 1991.

[51] W. Evans Wentz, The Tibetan Book of the Dead, Oxford Univ, London, 1968.

[52] Father Seraphim Rose, The Soul After Death, St. Herman of Alaska Brotherhood, Platina, 1988.

[53] J. A. Wheeler, "Law without law," in: J. A. Wheeler, W. H. Zurek, eds., Quantum Theory and Measurement Princeton Univ Press, Princeton, 1984, pp. 182-213.

[54] Č. Hadži-Nikolić, "Entheogenic shamanism: Anthropological category, transpersonal dimension or psychotherapeutic model," in: Ref. 3, pp. 351-354.

[55] M. Tomšić Akengen, "Abiku phenomenon: Spiritual origin and treatment of self-destructiveness," in: Ref. 3, pp. 344350.

[56] J. Vitale, I. Hew Len, Zero Limits: The Secret Hawaiian System for Wealth, Health, Peace, and More, Wiley, Hoboken, 2007.

[57] M. Kamp, Healing the Spiritual Way Through the Teachings of Bruno Gröning, Monchengladbach, Germany, 1999.

[58] A. Moorjani, Dying To Be Me: My Journey from Cancer, to Near Death, to True Healing, Hay House, Carlsbad, 2012.

[59] L. L. Hay, You Can Heal Your Life, Hay House, Carlsbad, 1984.

[60] http://www.dejanrakovicfund.org/2012_DRF_Stress_\&_Anti_Stress.pdf.

[61] Jon RG Turner, Private email communication, 17/01/2019.

[62] D. Raković, "Tesla's Inspired Holistic Questions of Creativity \& Education", Proc. 1st Int. Conf. Supervision in Psychotherapy, 22 June 2018, Center of Qualitative Research in Councelling and Psychotherapy, Timisoara, Romania, L-I. Vişcu, ed., Filodiritto Editore-Proceedings, Bologna, Italy (2018).

[63] D. Raković, "Tesla's Inspired Holistic Questions of Psychosomatics \& Spirituality", Proc. 2nd Int. Conf. Supervision in Psychotherapy, 28-29 June 2019, Center of Qualitative Research in Councelling and Psychotherapy, Timisoara, Romania, L-I. Vişsu, ed., Filodiritto Editore - Proceedings, Bologna, Italy (2019). 